PREPARED FOR THE U.S. DEPARTMENT OF ENERGY, UNDER CONTRACT DE-AC02-76-CHO-3073

PPPL-3045

PPPL-3045

UC-420,426

ALPHA PARTICLE LOSS IN THE TFTR DT EXPERIMENTS

BY

S.J. ZWEBEN, D.S. DARROW, H.W. HERRMANN, ET AL.

JANUARY 1995

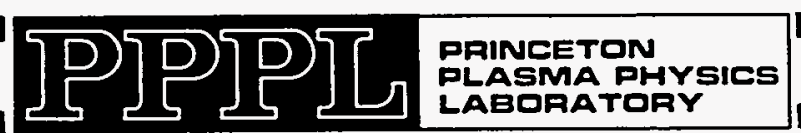

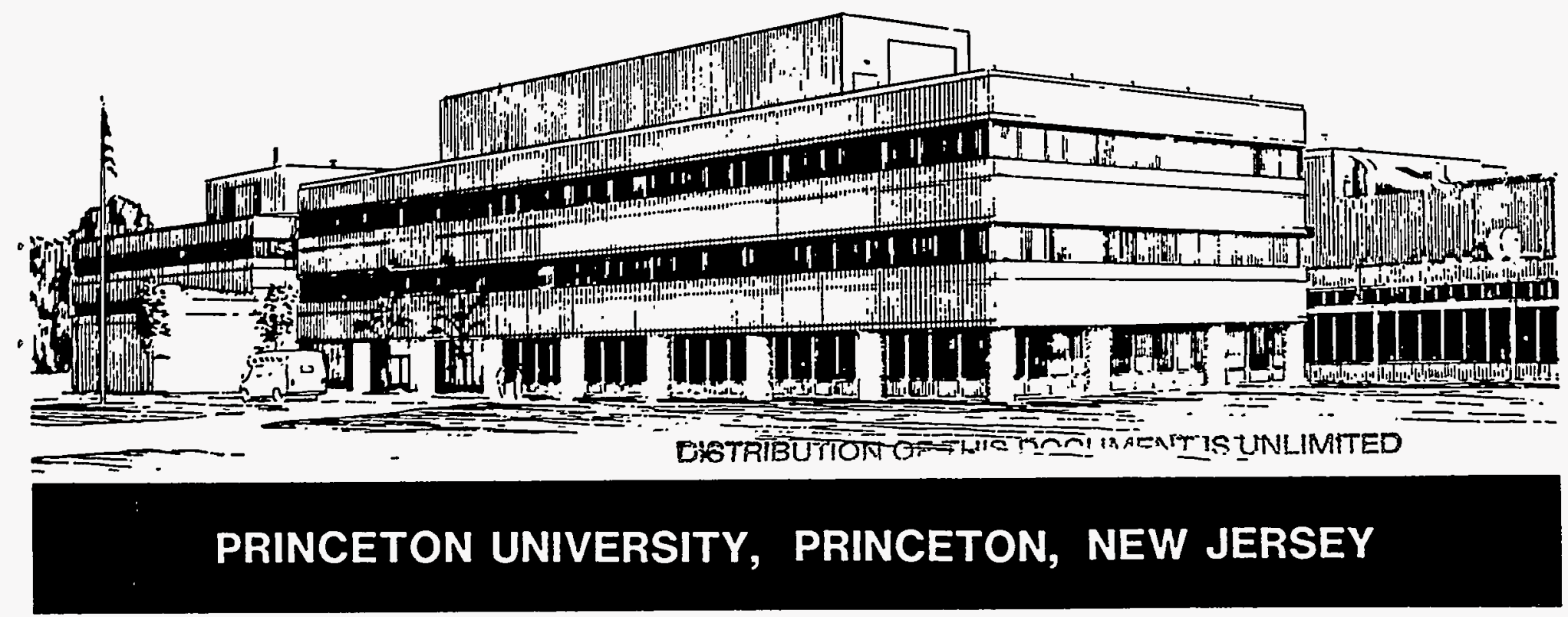




\section{NOTICE}

This report was prepared as an account of work sponsored by an agency of the United States Government. Neither the United States Government nor any agency thereof, nor any of their employees, makes any warranty, express or implied, or assumes any legal liability or responsibility for the accuracy, completeness, or usefulness of any information, apparatus, product, or process disclosed, or represents that its use would not infringe privately owned rights. Reference herein to any specific commercial produce, process, or service by trade name, trademark, manufacturer, or otherwise, does not necessarily constitute or imply its endorsement, recommendation, or favoring by the United States Government or any agency thereof. The views and opinions of authors expressed herein do not necessarily state or reflect those of the United States Government or any agency thereof.

\section{NOTICE}

This report has been reproduced from the best available copy. Available in paper copy and microfiche.

Number of pages in this report: 59

DOE and DOE contractors can obtain copies of this report from:

Office of Scientific and Technical Information

P.O. Box 62

Oak Ridge, TN 37831 ;

(615) 576-8401.

This report is publicly available from the:

National Technical Information Service

Department of Commerce 5285 Port Royal Road

Springfield, Virginia 22161

(703) $487-4650$ 


\section{DISCLAIMER}

Portions of this document may be illegible in electronic image products. Images are produced from the best available original document. 


\title{
Alpha Particle Loss in the TFTR DT Experiments
}

S.J. Zweben, D.S. Darrow, H. W. Herrmann, S. H. Bathał, R. V. Budny, C.S. Chang*, Z. Chang, E.D. Fredrickson, D.L. Jassby, L.C. Johnson, F. Levinton $\ddagger$ H. E. Mynick, D.K. Owens, J. Schivell, S. D. Scott, M. H. Redi, J. D. Strachan, K. Tobita\#, K.M. Young

Princeton Plasma Physics Laboratory, P.O. Box 451, Princeton, New Jersey 08543

† Fusion Physics and Technology

* Courant Institute, New York University, New York, 10012

\# JAERI, Naka, Japan

\begin{abstract}
Alpha particle loss was measured during the TFTR DT experiments using a scintillator detector located at the vessel bottom in the ion grad-B drift direction. The DT alpha particle loss to this detector was consistent with the calculated first-orbit loss over the whole range of plasma current $\mathrm{I}=0.6-2.7 \mathrm{MA}$. In particular, the alpha particle loss rate per DT neutron did not increase significantly with fusion power up to $10.7 \mathrm{MW}$, indicating the absence of any new "collective" alpha particle loss processes in these experiments.
\end{abstract}




\section{Introduction}

One alpha particle is created for each neutron in the fusion reaction $\mathrm{D}+\mathrm{T} \rightarrow \propto(3.5 \mathrm{MeV})+\mathrm{n}(14 \mathrm{MeV})$. A tokamak reactor will use the alpha particle power for heating, and will ignite only when the alpha power being transferred to the plasma balances the plasma energy loss rate. To insure ignition in a tokamak, the alpha particles must be well confined during their thermalization, e.g. for a timescale on the order of $\approx 1$ sec in ITER.

In an ideal, axisymmetric, MHD-quiescent tokamak the alpha particle confinement should be dominated by the finite orbit width of the alphas, which leads to "first-orbit" loss of the fat banana orbits. Globally, this loss is typically $<5 \%$ when the plasma current is above I=2 MA (see Appendix I), which should be sufficiently low for the purposes of alpha heating. However, a stricter constraint on alpha particle loss will be set by the need to protect the mechanical structures on the first wall of a reactor from potentially localized alpha heating [1]. Thus some understanding of the location of this alpha loss is needed to design the appropriate first-wall armor and cooling, even if the alpha loss is only few percent globally.

Previous tokamak experiments have simulated DT alpha particle confinement and loss using other fusion products such as the $1 \mathrm{MeV}$ triton from DD, or the fast ions used for auxiliary heating [2]. The usual conclusion from these experiments is that fast ions are generally well confined and thermalize "classically" in the absence of large MHD activity, i.e. the ion loss is determined solely by the static magnetic fields of the plasma current and toroidal field (TF) ripple. However, large MHD activity in TFTR and other tokamaks has also been shown to cause a fast ion loss fraction of up to $\approx 50 \%$, which would be intolerably large for a reactor. As discussed in the comprehensive review in Ref. 2, such MHD-induced fast ion loss can either be a "single particle" effect driven by the background plasma fluctuations (e.g. due to low $\mathrm{m} / \mathrm{n}$ kink-tearing modes), or a "collective" effect driven by the fast ions population itself (e.g. neutral beam driven TAE modes). One of the main motivations for the TFTR DT experiments was to see whether there was any new collective alpha-particle driven MHD activity would increase the alpha particle loss [3,4].

This paper presents the first detailed description of the TFTR alpha particle loss measurements made between the first DT experiments in Dec. 1993 and the achievement of 10.7 MW of DT fusion power in Nov. 1994. The measurements described here were made with a lost alpha detector located $90^{\circ}$ below the outboard midplane in the ion grad-B drift direction. The main result of this paper is that there was no substantial new collective alpha particle loss during these initial DT experiments. This is consistent with the observations that no new Alfven frequency fluctuation activity [5] or increased plasma losses [6] were observed in these DT plasmas. More detailed discussions of the results from the other alpha loss detectors $\left(60^{\circ}\right.$, $45^{\circ}$ and $20^{\circ}$ below the outer midplane), and of the occasional time-dependent MHD-induced alpha loss fluctuations, will be presented later. 


\section{Previous Experiments on Fusion Product Loss in TFTR}

A schematic picture of the lost alpha detector is shown in Fig. 1. The alpha detector element was a scintillator screen enclosed in a light-tight box located near the vessel wall, but well outside the plasma. The detector used here was located $90^{\circ}$ poloidally below the outer midplane at a toroidal location between two TF coils. It was fixed in the vessel with its aperture on a field line $<1 \mathrm{~cm}$ radially outward from the nearest poloidal limiter (after taking into account the small field line movement due to TF ripple). This was close enough to avoid any shadowing of the escaping first-orbit alpha loss by this limiter, which was $\approx 2 \mathrm{~m}$. away toroidally.

The pinhole/slit aperture pair shown in Fig. 1 allowed fast ions with gyroradii in the range $\approx 2-12 \mathrm{~cm}$ to hit the scintillator screen, resulting in a visible light image which was optically transferred to an intensified CCD camera using a quartz lens and coherent fiberoptic bundle underneath the vessel. The "pinhole" entrance aperture dimensions are $\approx 1 \mathrm{~mm}$ high $\times 2$ $\mathrm{mm}$ wide, while the slit was $1 \mathrm{~mm}$ high $\times 1.5 \mathrm{~cm}$ wide, $1 \mathrm{~cm}$ behind the pinhole. A $3 \mu \mathrm{m}$ aluminum foil behind the slit sets the lower limit of detectable alpha energy to be $\approx 1 \mathrm{MeV}$. The hardware and calibration of this system have been described elsewhere [7-10].

The 2-D scintillator light emission images were analyzed and interpreted using a detector simulation code which calculates the expected scintillator images of fast ions incident at a given pitch angle and gyroradius, including the geometrical and optical resolutions of the system [11]. The pitch angle is defined here with respect to the local toroidal field $\mathrm{B}$, with $90^{\circ}$ being perpendicular to $\mathrm{B}$, and $0^{\circ}$ being along the co-I (plasma current) direction, and so is a measure of the ion's magnetic moment at a given gyroradius. The alpha gyroradius as defined here is $\rho=(2 E / M) 1 / 2 / \Omega$, where $E$ is the energy of an alpha with mass $M$ and gyrofrequency $\Omega$, i.e. the gyroradius is a measure of the ion's energy. Typically $3.5 \mathrm{MeV}$ alpha particles have a gyroradius of $\rho \approx 5 \mathrm{~cm}$ at the $90^{\circ}$ detector for the nominal $\mathrm{B}=5 \mathrm{~T}$ toroidal field of TFTR (of course, this gyroradius varies with the local $\mathrm{B}$ at the detector).

This system has been used for several years to measure the loss of DD fusion products ( $3 \mathrm{MeV}$ protons and $1 \mathrm{MeV}$ tritons), which have gyroradii only $\approx 10 \%$ less than the $3.5 \mathrm{MeV}$ alphas from DT. Previous results can be summarized as follows: (a) for MHD-quiescent $R=2.6 \mathrm{~m}$ plasmas the loss to the $90^{\circ}$ detector was consistent with the expected first-orbit loss over the plasma current range $\mathrm{I}=0.6-2.0 \mathrm{MA}[11]$, (b) an additional anomalous delayed loss was seen at $90^{\circ}$ for $R=2.45 \mathrm{~m}$ plasmas at $\mathrm{I}=1.4-2.5 \mathrm{MA}$ [12], (c) during large coherent MHD activity there could be up to a factor of three increase in the loss at the $90^{\circ}$ and $20^{\circ}$ detectors [13], (d) the pitch angle distribution of the loss at $60^{\circ}$ and $45^{\circ}$ agreed reasonably well with the firstorbit model at I=1.2 MA [14], and (e) there was a large non-first-orbit loss component in the $20^{\circ}$ detector which was consistent with the expected loss 
from stochastic TF ripple diffusion. [15]. The remaining anomalous features of these "single-particle" DD loss measurements were summarized recently to help isolate any new "collective" effects in the DT experiments [16].

Between the DD run in 1992 and the DT run in 1993 there were several changes to this lost alpha diagnostic. Based on measurements at the Los Alamos Van de Graaff accelerator [9], the scintillator material was changed from zinc sulfide (P11 or P31) to yttrium aluminate (P46), which was $\approx 20$ times less efficient, in order to guarantee the linearity and damage resistance of the phosphor at the $\approx 100$ times larger ion flux levels expected in DT (estimated to be $\approx 10^{10}$ alphas $/ \mathrm{cm}^{2}$ on the scintillator for $\approx 1000 \mathrm{DT}$ shots). In addition, the intensified video camera which recorded the light from the scintillators was moved to a shielded enclosure in the basement to avoid direct neutron noise (none was observed in DT) and additional shielding was added around the coherent quartz fiberoptic bundle under the vessel to minimize neutron-induced background light generated in the quartz (some was still seen) [10].

\section{Modeling of Alpha Particle Loss to the $90^{\circ}$ Detector}

Calculations of the local first-orbit loss to the $90^{\circ}$ detector were made using an axisymmetric Lorentz code which calculated the exact trajectory of the alpha particle from the detector backwards into the plasma, including its finite gyroradius and gyrophase [17]. Using this code, it was found that the first-orbit alpha loss $90^{\circ}$ below the outboard midplane depends mainly on the plasma current, which determines the displacement of the alphas from the flux surfaces of their birth.

Calculations of the global first-orbit and toroidal field (TF) ripple loss of alphas are not used for interpreting the localized experimental results in this paper, and so are summarized separately in Appendix I. However, one important result of these calculations is that $\mathrm{TF}$ ripple-induced alpha loss is not expected at the $90^{\circ}$ detector location, since the confined trapped alpha orbits which are affected by TF ripple are lost mainly near the outer midplane. First-orbit loss is the dominant classical loss process at the $90^{\circ}$ detector location.

Typical first-orbit alpha loss trajectories to the $90^{\circ}$ detector as calculated by the Lorentz code are shown in Fig. 2. The $q(r)$ profiles for these analyses were taken from the TRANSP (time-dependent) transport code, which were checked with the measured $q(r)$ profiles from the Motional Stark Effect (MSE) diagnostic, as described in Appendix II. The orbits shown in Fig. 2 are calculated "backwards in time" from the detector to the plasma midplane for three different pitch angles at two different plasma currents. 
At the lowest current of I=0.6 MA the 90 detector can "see" alpha loss orbits from the whole alpha source profile, including the high source rate region near the plasma center. Therefore the total alpha loss at $90^{\circ}$ was found to be relatively insensitive to the $\mathrm{q}(\mathrm{r})$ or alpha (neutron) source profiles. Thus the alpha loss at I=0.6 MA was used as an in situ calibration of the loss at higher currents.

For the I=2.0 MA case shown in Fig. 2 there was no first-orbit loss to the $90^{\circ}$ detector from within the high fusion source rate region near the plasma center. The largest component of first-orbit loss was from orbits near the "fattest" banana at a pitch angle of $X=60^{\circ}$, measured with respect to the local toroidal direction at the detector. Therefore the total first-orbit alpha loss to the $90^{\circ}$ detector at this current does depend on the assumed current profile, which determines the shape of the fattest banana orbit, and also on the alpha source profile near the orbit's closest approach to the plasma center.

This Lorentz code calculates the expected first-orbit alpha loss at a given pitch angle by calculating the alpha orbit, integrating the alpha source function along this loss orbit, and correcting for the area, solid angle, and orientation of the specific detector aperture pair [11]. The total "alpha collection fraction" is defined here as the ratio between the alpha loss into the $90^{\circ}$ detector (i.e. integrated over the aperture's pitch angle and gyroradius acceptances) and the global alpha source rate. Thus the alpha collection fraction for this detector was typically $\approx 10^{-8}-10^{-9}$, the scale of which is mainly set by the small size of the pinhole aperture $\left(.02 \mathrm{~cm}^{2}\right)$ with respect to the area of the bottom of the TFTR vessel $\left(\approx 5 \times 10^{5} \mathrm{~cm}^{2}\right)$.

\section{Alpha Loss to the $90^{\circ}$ Detector}

Most of the previous analysis of DD fusion product loss in TFTR was done with data from the detector $90^{\circ}$ below the midplane [11-14]. This was mainly because this detector has the best optical coupling efficiency between the scintillator and the fiberoptic bundle, and so had the largest signal levels and the best resolution of the three fixed detectors. Also, since no TF ripple loss was expected at $90^{\circ}$ (see Appendix I), the analysis of the expected loss to this detector was relatively straightforward. The DT results are described in Secs. 4.1-4.8, with a summary of the corresponding DD results in Sec. 4.9.

\subsection{Plasma Parameters}

All of the discharges discussed in this paper occurred during the 1993-94 TFTR run period, which included both DT and corresponding DD discharges for comparison. All these plasmas had the same major and minor radii of $R=2.52 \mathrm{~m}, \mathrm{a}=0.87 \mathrm{~m}$, and a toroidal field in the narrow range 
of $\mathrm{B}=4.8-5.1 \mathrm{~T}$. Neutral beam injection was used with powers of 5-40 MW for 0.7-1.0 sec in these "supershots" (no ICRH was not used here).

Table 1 summarizes the main parameters of this data set, sorted according to their plasma current. The $40 \mathrm{DT}$ discharges came from four types of experiments: (1) experiments at $I=2.0 \mathrm{MA}$ with a small tritium fraction $(\approx 2 \%)$ in all the neutral beam injectors (NBI), (2) a plasma current scan over I=0.6-1.8 MA with a single tritium beam source, i.e. 2.5 MW of T beams, (3) an alpha heating experiment at $I=1.8 \mathrm{MA}$ and $\approx 20-25 \mathrm{MW}$ of $50 / 50$ "full DT", and (4) fusion power experiments at $I=2.0,2.5$, and.2.7 MA with up to $40 \mathrm{MW}$ of full-DT $[5,6]$. One of these discharges had the highest sustained fusion power and alpha pressure of any DT shot to data; the alpha parameters calculated by TRANSP for this shot (\#76770) are summarized in Table 2. One of these discharges also had the highest instantaneous fusion power to date, i.e. 10.7 MW (\#80539).

At the higher plasma currents $I \geq 1.8 \mathrm{MA}$, the radial profiles of the magnetic safety factor $q(r)$ and the fusion reaction rate $S(r)$ are important in determining the local first-orbit loss rate (see Sec. 3). For the first-orbit loss calculations in this paper, the $\mathrm{q}(\mathrm{r})$ and $\mathrm{S}(\mathrm{r})$ profiles were taken from timedependent TRANSP simulations, rather than from direct measurements, since these profiles were measured on only a subset of the shots for which lost alpha data was available. However, the TRANSP profiles agreed well when cross-checked with the $q(r)$ measurements from MSE and $S(r)$ measurements from the neutron collimator, as described in Appendix II.

\subsection{Lost Alpha Signals and Backgrounds}

Data on alpha loss was taken for all of the DT discharges in the initial DT run of Dec. 1993. The raw scintillator data from two of the first $\mathrm{DT}$ shots at $\mathrm{I}=0.6 \mathrm{MA}$ and $\mathrm{I}=2.0 \mathrm{MA}$ is shown in Fig. 3. This data was averaged over the near-steady-state time $0.4-0.7 \mathrm{sec}$ after the start of NBI, i.e. 3.0-3.7 sec after the start of the plasma current. The I=0.6 MA shot had $5 \mathrm{MW}$ of NBI (50/50 D/T), while the I=2.0 MA shot had $29 \mathrm{MW}$ of NBI (40/60 $\mathrm{D} / \mathrm{T}$ ). The latter was the discharge with the maximum fusion power of 6.2 MW during the initial DT run period at $I=2.0 \mathrm{MA}[5,6]$.

The height coordinate in these 3-D plots is proportional to the signal intensity as measured by the CCD camera, the output of which is linear with light intensity over the range from $\approx 45$ units (black level) to $\geq 200$ units (out of 256). The elevated square region covering most of the pixel vs. line plane corresponds to the quartz fiberoptic bundle, which emits visible light in the same wavelength range as the scintillator $(\approx 550 \pm 35 \mathrm{~nm})$, due to the neutron and gamma induced fluorescence $[10,18]$. The lost alpha signals are the localized peaks within this background. It is clear that the alpha signal/neutron background ratio is larger for the $I=0.6 \mathrm{MA}$ case than for the I=2.0 MA case, directly indicating a larger alpha collection fraction at the lower current, as expected. 
These raw CCD images are interpreted using the scintillator "map" shown in Fig. 4. The grid of pitch angle vs. gyroradius coordinates was calculated for incident alpha particles using a detector simulation code [14], which includes the energy attenuation in the $3 \mu \mathrm{m}$ aluminum foil (typically $\approx 20 \%$ at $3.5 \mathrm{MeV}$, with a cutoff at $\approx 1 \mathrm{MeV}$ ) and the geometrical spread allowed by the finite aperture sizes. The scintillator position with respect to the quartz bundle was determined by an in-vessel calibration before the DT run [10]. Note that these grid points represent only the centroids of the impact patterns for alphas of a given pitch angle and gyroradius; however, the full impact distribution and the optical resolutions are taken into account in interpreting the pitch angle and gyroradius distributions (Secs. 4.7-4.8).

The camera data is analyzed by subtracting the brightness of the background region of the image from the image as a whole. The background subtraction region was within the boundary of the scintillator image, but outside the region of the alpha signal, as indicated by the gray region in Fig. 4. The image was then unfolded using the scintillator map of Fig. 4 into a pitch angle vs. gyroradius $(X, \rho)$ distributions, such as shown in Fig. 5 for data of Fig. 3. The total lost alpha signal level is found by integrating over the $(X, \rho)$ coordinates shown in Fig. 4 , i.e. $X=45^{\circ}-90^{\circ}$ and $\rho=3.5-10 \mathrm{~cm}$, which includes essentially all of the lost alpha signal for all plasma currents, and then subtracting out the level in the background region separately for each time frame.

The signal levels in the background region of the camera field scaled linearly with the standard neutron rate monitors when integrated over 0.4$0.7 \mathrm{sec}$ after the start of NBI (within $\approx 10 \%$ ), as expected from independent measurements of the fiberoptic fluorescence [18]. The time dependence of the signal level within the whole $(X, \rho)$ map of Fig. 4 was simultaneously monitored using a photomultiplier tube, and for high current discharges it closely followed the neutron rate vs. time. This was expected since at high currents $\approx 3 / 4$ of the total signal within the whole map was due to the neutron background (see Fig. 3).

However, the background signal level as determined from the camera images had an instrumental delay of $\approx 50-100 \mathrm{msec}$ with respect to the standard neutron signals (a similar delay was seen in the camera's response to an optical test pulse anywhere in the field of view). For the data in this paper the time dependence of the alpha loss signal not corrected for this delay, but instead the time dependence of the alpha loss signal was compared with the time dependences of the fiberoptic neutron background signal (within the gray area of Fig. 4). This procedure automatically corrects for this hardware delay and insures accurate cross-timing between the alpha loss and neutron signals.

The time dependence of the net alpha loss signal for a typical DT discharge is compared with that for an equivalent DD discharges in Fig. 6. Both these discharges had I=2.0 MA and 27 MW of NBI between 3.0 and 3.7 
sec (\#73235, 73236), and the lost alpha detector camera gains and data analysis procedures were exactly the same for both. The total loss signal within the $(X, p)$ grid during the DD shot was $<5 \%$ of the signal during the DT shot (after the appropriate background subtractions); therefore essentially all of the signal during the DT shot was due to alpha particle loss, and not to DD fusion product loss.

The magnitude of the DT alpha loss rates cannot be directly compared with the DD fusion product loss rates, since the scintillator light output from $1 \mathrm{MeV}$ tritons has not been directly measured $[9,10]$. However, at I=0.6 MA, where the DT and DD fusion product loss fractions should be similar, the measured scintillator light output per neutron for DT vs. DD discharges was $\approx 1.5 \pm 0.3$, i.e. the signal levels during DT were $\approx 100$ times larger than during similar DD discharges, mainly due to the $\approx 60$ times larger neutron flux in DT. This is roughly consistent with the expected light output ratio, since only a fraction of the $3 \mathrm{MeV}$ proton energy is deposited within these thin P46 scintillators. Thus the measured alpha loss signal was close to that expected from the previous DD measurements. The absolute calibration for DT is discussed in Sec. 4.6.

\subsection{Time Dependence}

In Fig. 7 the time dependence of the lost alpha signal is compared with the time dependence of the neutron background signal for three typical DT shots. The alpha loss signals are the averages within the $(x, p)$ grid of Fig. 4, after subtraction of the neutron-induced fiberoptic background level (gray area of Fig. 4). The "neutron background" traces in Fig. 7 are the signal levels within the "gray area" shown in Fig. 4, which is dominated by the fiberoptic fluorescence, which can be assumed to be proportional to the DT neutron rate (see Sec. 4.2). These two signals are normalized to each other at their peaks to show their relative time behavior more clearly.

The total alpha loss rate for the single source tritium shot at $I=0.6$ MA followed closely the neutron rate vs. time, as it did for all other low-tomoderate powered low current DT shots (I $\leq 1.4 \mathrm{MA}$ ). However, for the moderate-powered single tritium source shot at I=1.8 MA, and for the highpowered full DT shot at I=2.0 MA (which was the discharge with the maximum fusion power at this current), there was some difference between time dependences of the alpha loss and neutron background during and after NBI.

These differences are shown more clearly in Fig. 8, which compares the time dependence of the alpha loss signal normalized by the neutron background signal to the time dependence of the calculated first-orbit alpha collection rate for the discharges of Fig. 7. These calculated alpha collection rates were based on TRANSP analyses of the profile shapes vs. time, and were normalized to the data at $0.6 \mathrm{sec}$ after the start of NBI. For all cases the measured normalized alpha loss was within about $\pm 20 \%$ of the 
calculated first-orbit loss rate. However, given the estimated experimental uncertainty (shown by the error bars), it is not possible to isolate any real discrepancies between the data and the model. The uncertainties are discussed in more detail in Sec. 4.6.

The conclusion from these and other DT discharges of Table 1 is that the time dependence of the alpha loss signals is roughly consistent with the expected prompt first-orbit loss, i.e. approximately proportional to the DT neutron rate during a single discharge at a constant current. There was no sign of any significant MHD-induced losses during these DT discharges, such as previously seen as a sudden increase in the ratio of the fusion product loss to the neutron rate in $\mathrm{DD}$ (see $\mathrm{Sec} 6.3$ ). There was also no sign of the "delayed loss" seen previously in DD (see Sec. 4.9).

\subsection{Neutron Rate Dependence}

Any systematic increase in the alpha loss rate with an increasing DT neutron rate could indicate some new "collective" alpha loss mechanism, since the confined alpha population (which presumably would cause such an effect) should increase with the DT rate. This dependence of the alpha loss on the neutron rate should be examined at a constant plasma current, since then the "single-particle" first-orbit alpha loss rate should be nearly independent of the neutron rate.

Figure 9 shows the neutron-normalized alpha loss to the $90^{\circ}$ detector vs. the peak DT fusion power for all of the high-current discharges in the data set of Table 1. The alpha loss was averaged over the near-steady-state period 0.4-0.7 sec after the start of NBI, and was normalized to the standard neutron rate signals integrated over that time. The resulting alpha lost rates per DT neutron were further normalized to the calculated "alpha collection fraction" for the $90^{\circ}$ detector at I=0.6 MA, as explained in Sec. 4.5 below. This allows the expected first-orbit levels to also be shown in Fig. 9.

The result was that there was no significant increase in the lost alpha collection fraction with increasing DT fusion power up to the maximum of $10.7 \mathrm{MW}$, which corresponded to $\approx 2 \mathrm{MW}$ of alpha source power. At $I=2.0 \mathrm{MA}$ the range of $\mathrm{DT}$ neutron rates covered a factor of $\approx 40$, where the lowest alpha source rates came from trace-tritium shots with DT rates similar to their $\mathrm{DD}$ neutron rate (the contribution from $\mathrm{DD}$ fusion product loss was subtracted out for these cases). At I=2.7 MA the range of DT rates was due to different beam and plasma performance in full DT. The alpha loss in the at I=2.7 MA discharge with the maximum DT rate of $3.6 \times 10^{18} \mathrm{n} / \mathrm{s}$ was evaluated near the peak of its fusion power $(3.7-3.75 \mathrm{sec})$, instead of averaged over 0.4-0.7 sec after the start of NBI, since the neutron rate changed significantly during this time. The calculated first-orbit alpha collection fraction at this time in this discharge was $3.1 \times 10^{-9}$, which is $\approx 15 \%$ below the averaged first-orbit loss for this current shown in Fig. 9 . 
The conclusion from this analysis is that the measured alpha collection fraction did not show any signs of collective alpha loss with increased DT rate, and that the results were approximately consistent with the expected first-orbit loss. Given the uncertainties in the data and calculations (see Sec. 4.6), the shot-to-shot variations of $\approx 20 \%$ in the measured alpha loss rate vs. neutrons could not be convincingly correlated with the shot-to-shot variations of $\approx 10-15 \%$ in the calculated first-orbit loss collection fraction around these averaged levels.

\subsection{Plasma Current Dependence}

The plasma current dependence of the neutron-normalized alpha loss is shown in Fig. 10 for all the DT discharges of Table 1. Each "x" represents a different discharge for which the loss signals are integrated over the $(X, \rho)$ map of Fig. 4 during the near-steady-state period 0.4-0.7 sec after the start of NBI. The neutron background has been subtracted out, as discussed in Sec. 4.3, and the alpha loss signals have been normalized to the standard neutron rate signals averaged over this time.

This data is further normalized to the calculated first-orbit alpha collection fraction at I=0.6 MA, at which point the first-orbit loss is expected to dominate over any other loss process. This calculated alpha collection fraction is defined as the first-orbit alpha loss rate passing through the detector's apertures onto the scintillator, divided by the total DT neutron (alpha) source rate. For example, an I=2.0 MA discharge with $\approx 2 \times 10^{18} \mathrm{DT}$ neutron/sec with an alpha collection fraction of $\approx 5 \times 10^{-9}$ would have $\approx 10^{10}$ alphas/sec passing through the apertures and hitting the scintillator. This normalization of the data to the first-orbit loss calculation at $\mathrm{I}=0.6 \mathrm{MA}$ provides an in situ calibration of the alpha loss at higher currents (see also Sec. 4.6).

The whole DT data set was essentially consistent with the first-orbit alpha loss model calculations indicated by the shaded region in Fig. 10. This implies that there was no substantial anomalous alpha particle loss seen in the $90^{\circ}$ detector for this data set. Although the data of Fig. 10 refer to time-averaged alpha loss over a $0.3 \mathrm{sec}$ interval near the peak of the DT neutron emission, there was also little or no evidence for fluctuating MHDinduced loss in these discharges.

The first-orbit model region shown in Fig. 10 is based on the Lorentz code analysis for several discharges at each current (see Sec. 3). The neutron source and plasma current profiles used for these calculations were taken from the time-dependent TRANSP analysis of these discharges at $0.6 \mathrm{sec}$ after the start of NBI. Similar analyses made earlier using the profiles from the time-dependent SNAP transport code gave a somewhat differently-shaped model prediction, as shown previously by the lower edge of the shaded region in Fig. 4 of Ref. 5 (the TRANSP-based calculations were shown by the upper edge of the shaded region in that figure are 
similar to the TRANSP calculations shown here). The profiles from the TRANSP code are considered to be more accurate, and have been checked against the measured profiles, as discussed in Appendix II.

The scatter apparent in the data at a given plasma did not show any systematic increase with DT neutron rate, as discussed in the previous section. The largest discrepancy between the data and the first-orbit loss modeling was at the highest plasma current of $I=2.7 \mathrm{MA}$, where the signalto background ratio was the smallest and the experimental uncertainty was largest. The uncertainties in the measured and calculated alpha losses are discussed in more detail in Sec. 4.6. The conclusion from that analysis is that the agreement between experiment and theory at $I \approx 2.0 \mathrm{MA}$ is good to within a joint uncertainty of $\pm 50 \%$, when based on the in situ calibration at $\mathrm{I}=0.6 \mathrm{MA}$.

\subsection{Uncertainties and Absolute Calibration}

Figs. 8-10 showed good agreement between the measured alpha loss in the $90^{\circ}$ detector and the calculated first-orbit alpha loss. However, there were several uncertainties not shown explicitly in those figures which are discussed below, along with the absolute calibration.

\subsubsection{Uncertainties in the Measurements}

The measurements of the neutron-normalized alpha loss vs. plasma current as summarized in Fig. 10 showed a $\pm 5-10 \%$ scatter at $I \leq 1.4 \mathrm{MA}$, but up to a $\pm 30 \%$ scatter at $\mathrm{I}=1.8-2.7 \mathrm{MA}$. At the higher plasma currents, there were also time variations in the normalized alpha loss of $\pm 10-20 \%$ during 0.4-0.7 sec after the start of NBI, as shown in Fig. 8. Since there was little or no systematic variation with the DT neutron rate, as shown in Fig. 9, this scatter is most likely due either to a variation in the first-orbit loss due to plasma profile changes (see Sec. 4.6.2), or to uncertainties in the evaluation of the measured alpha loss.

The main experimental uncertainty in these measurements was due to the background subtraction processes (Sec. 4.3). For example, at a current of $I=2.0 \mathrm{MA}$, the peak signal-to-neutron-background ratio was only about 2.5:1 (see Fig. 3), and the average signal to background level within the whole $(\rho, \chi)$ map (over which the analysis was made) was $\approx 1: 3$. Thus if the average background level within the $(\rho, X)$ map was only $\approx 10 \%$ different than the average level within the "background region" of the scintillator (see Fig. 4), there would be an $\approx 30 \%$ systematic error in the inferred alpha signal level at this current. This error cannot be easily evaluated, since the shape of the fiberoptic background pattern can not be measured without the presence of alpha loss. However, this type of error would change the inferred alpha loss collection fraction by some constant number over the 
whole range of plasma currents, and so would not significant change the shape of the inferred alpha loss vs. current plot in Fig. 10.

Other measurement uncertainties include the drift in the camera gain vs. time, which was monitored daily and was $<10 \%$ over the duration of these experiments, and a shot-to-shot uncertainty of $\approx 5 \%$ in the epithermal DT neutron measurement used for normalization [19]. The fiberoptic transmission was monitored by a test fiber close to the signal fibers, and showed a negligible change in both transient and permanent opacity over this experimental run. Overall, the neutron-normalized alpha loss measurements for the full DT shots at $\mathrm{I} \approx 1.8-2.7 \mathrm{MA}$ are uncertain by an estimated $=30 \%$ with respect to the alpha loss at I=0.6 MA, which provides an in situ calibration.

\subsubsection{Uncertainties in the Calculations}

The calculated alpha first-orbit collection fraction at $90^{\circ}$ at I $=0.6 \mathrm{MA}$ had a very small variation over the three DT shots in Table $1(\leq 5 \%)$. These variations were typically higher at the higher currents of $\mathrm{I}=1.8-2.7 \mathrm{MA}$ (i.e. $\pm 10-15 \%)$ due to the increased sensitivity to plasma current and neutron source profiles, as discussed in Sec. 2. These calculations used the profiles from the TRANSP code at $0.6 \mathrm{sec}$ after the start of NBI.

The profiles from TRANSP were spot-checked with measurements of $\mathrm{q}(\mathrm{r})$ and $\mathrm{S}(\mathrm{r})$ for some of the discharges in this data set, as described in the Appendix II. The $90^{\circ}$ alpha collection fraction calculated using the measured $\mathrm{q}(\mathrm{r})$ profiles were within $\pm 10 \%$ of those using the TRANSP $\mathrm{q}(\mathrm{r})$ profiles over I=0.6-1.8 MA, and the alpha loss calculated for an $I=1.8 \mathrm{MA}$ discharge using the measured neutron profile was within $\approx 15 \%$ of that calculated using TRANSP neutron profile. There was also an $\approx 10-20 \%$ uncertainty in these calculations due to imprecise knowledge of three aperture dimensions which determine the alpha flux into the detector. Overall, the calculated first-orbit alpha loss at the highest currents $I \geq 1.8$ MA has an estimated uncertain of $\pm 30 \%$.

In summary, the measured alpha loss for the high current, high power full DT shots agrees with the calculated loss to within a joint uncertainty of less $\approx 50 \%$ (i.e. $\approx 30 \%$ each from experiment and calculation), when normalized to the reference discharges at $\mathrm{I}=0.6 \mathrm{MA}$, which serve as an in situ calibration. This is approximately the same uncertainty found previously in comparing measured and calculated loss for DD fusion products in the $90^{\circ}$ detector [11].

\subsubsection{Absolute calibration}

An independent absolute calibration of the total lost alpha flux onto the scintillator was also made by exposing a sample P46 scintillator to a 
beam of 3.5 MeV alphas at the Los Alamos Van de Graaff accelerator [9,10]. The scintillator light due to an absolutely calibrated alpha beam was compared with the light from a portable calibration lamp, which was then transferred into the TFTR vessel and mounted at the scintillator locations during a machine opening.

The absolute alpha collection fraction estimated in this way for the $\mathrm{I}=0.6 \mathrm{MA}$ discharges was $\approx 1.4 \times 10^{-8}$, which was close to the calculated firstorbit loss fraction of $\approx 2.2 \times 10^{-8}[10]$. The absolute calibration was estimated to have a $\pm 60 \%$ uncertainty, i.e. $\approx 50 \%$ from the measurements at the. Van de Graaff, and $\approx 25 \%$ from the calibration lamp. Thus the measured alpha loss in TFTR agreed with the absolute calibration to within their joint uncertainties. The data of Figs. 9 and 10 were normalized to the calculated first-orbit loss at I=0.6 MA due to the relatively large uncertainty in this absolute calibration.

\subsection{Pitch Angle Dependence}

The first-orbit loss model predicts that the alpha loss should be peaked near the pitch angle of the "fattest" banana orbit which passes closest to the high source rate region near the plasma center. This pitch angle should also decrease systematically with increased plasma current, as illustrated in Fig. 2. Anomalous loss of DD fusion products has previously been noticed by changes in these pitch angle distributions. For example, the "delayed loss" was seen at an unusually high pitch angle [12], and MHD-induced loss was sometimes localized near the passing/trapped boundary [16].

The pitch angle distributions of alpha loss in the single tritium source shots in the plasma current range $\mathrm{I}=0.6-1.8 \mathrm{MA}$ are shown in Fig. 11. The shapes of these distributions are quite reproducible from shot-toshot, and agree well with those calculated from the first-orbit loss code. The curves shown take account of the $\approx 5^{\circ}$ optical resolution and $\approx 3^{\text {o }}$ geometrical resolution of the detector system, and the measurements were absolutely calibrated with respect to pitch angle by in-vessel alignments to within $\approx 3^{\text {g}}$. The modeling does not yet account for the geometrical cut-off in the pitch distribution above $\approx 83^{\circ}$, so tends to overestimate the loss at high pitch angles at the lowest plasma currents.

The pitch angle distributions of the measured alpha loss for two of the higher current I=2.0 MA shots are shown in Fig. 12. One of these shots was a single tritium source shot with a relatively low DT reaction rate (\#73234), and the other was the shot with the maximum fusion power of 6.2 MW at I=2.0 MA (\#73268). The measured and calculated pitch angle distributions are similar to each other and to the first-orbit model calculations, indicating the absence of any new alpha loss mechanism in the discharge with the highest confined alpha population at this current. 


\subsection{Gyroradius Dependence}

The first-orbit loss model predicts that alphas will be lost near their birth energy of $\approx 3.5 \mathrm{MeV}$, with a Doppler spread of up to $\pm 0.5 \mathrm{MeV}$ caused by the beam-target and beam-beam reactions (which contribute $\approx 75 \%$ to the fusion reactivity). The alpha loss orbits and loss fractions do not change significantly within this energy range, so the alpha orbits throughout this paper were calculated by assuming an alpha energy of exactly $3.5 \mathrm{MeV}$ (in alpha confinement terms, the maximum spread of $\pm 0.5 \mathrm{MeV}$ is equivalent to only a $\pm 7 \%$ change in plasma current).

The gyroradius distributions of the measured alpha loss for the single tritium source shots in the current scan from I=0.6-1.8 MA are shown in Fig. 13. These distributions are plotted vs. the gyroradius centroid coordinate along the scintillator map shown in Fig. 4. There is a considerable spread in this direction due to the finite aperture sizes and optical resolutions, so that a monoenergetic $3.5 \mathrm{MeV}$ alpha distribution is not expected to be well localized at its corresponding $\rho=5.4 \mathrm{~cm}$ for these cases. The modeled gyroradius distributions for $3.5 \mathrm{MeV}$ alphas are also shown in Fig. 13, including these spreads as calculated by the detector simulation code. The shapes of the experimental distributions are quite reproducible and agree fairly well with the model for $3.5 \mathrm{MeV}$ alpha loss. The small differences at large and small $\rho$ are most likely are due to uncertainties in the background subtraction or modeling. Note that the measured distributions were absolutely calibrated by an in-vessel alignment to within $\approx 1 \mathrm{~cm}$ in this gyroradius centroid coordinate.

The gyroradius distributions of the measured alpha loss for two of the $\mathrm{I}=2.0 \mathrm{MA}$ shots are shown in Fig. 14. One of these shots was a single tritium source shot with a relatively low DT reaction rate (\#73234), and the other was the shot with the maximum fusion reactivity at this current (\#73268). The measured gyroradius distributions are similar to each other, and are fairly well fit by the $3.5 \mathrm{MeV}$ alpha loss model. The model curves for 2.5 MeV and 4.5 MeV alphas are also shown for comparison, normalized at a fixed peak height.

The conclusion from this analysis is that the energy distribution of the measured alpha loss in the $90^{\circ}$ detector is independent of the DT reaction rate, and roughly consistent with the expected first-orbit loss distribution (i.e. at gyroradii corresponding to alphas at $3.5 \pm 0.5 \mathrm{MeV}$ ). These gyroradius distributions are also independent of time, similar to previous for DD fusion product measurements [11].

However, the presence of a comparable level of lower-energy alpha loss components at $\approx 2 \mathrm{MeV}$ can not be excluded, given the present uncertainties in the modeling and data. Also, it should be noted that the presence of alpha loss at energies below $\approx 1 \mathrm{MeV}$ can not be determined at all from this detector, due to the presence of the foil behind the slit aperture of this detector (see Fig. 1). 


\subsection{Comparison With DD Fusion Product Loss}

The loss of DD fusion products in TFTR ( $3 \mathrm{MeV}$ protons and $1 \mathrm{MeV}$ tritons) has previously been analyzed only for plasmas with $R=2.62 \mathrm{~m}$ and $\mathrm{R}=2.45 \mathrm{~m}$. For MHD-quiescent plasmas at $\mathrm{R}=2.6 \mathrm{~m}$ the loss at the $90^{\circ}$ detector was consistent with first-orbit loss [11], but there was an additional delayed loss present at $R=2.45 \mathrm{~m}$ [16]. The DT discharges described in this paper had a different major radius of $R=2.52 \mathrm{~m}$ (to optimize performance); therefore, the corresponding DD discharges will be described here explicitly. At least one DD comparison shot was made prior to each full DT discharge to avoid DT contamination, as listed in Table 1 (except for $I=2.7$ MA).

The patterns of pitch angle vs. gyroradius for DD fusion product loss at $90^{\circ}$ are shown in Fig. 15 for $\mathrm{I}=0.6 \mathrm{MA}$ and $\mathrm{I}=2.0 \mathrm{MA}$ discharges, analyzed similarly to the corresponding DT cases in Fig. 5. The DD patterns are basically similar to those for DT. The DD pitch angle distributions as shown in Fig. 16 also agree well with the calculated first-orbit loss distribution, which is not expected to vary significantly between DD and DT fusion products. The DD gyroradius distributions shown in Fig. 17 have a small but systematic downward shift in the gyroradius distribution compared with similar DT discharges, which is consistent with the expected $\approx 10 \%$ smaller gyroradius of DD fusion products compared with DT alphas.

The largest difference between the DD and DT fusion product loss in the $90^{\circ}$ detector was a somewhat larger loss at high pitch and low gyroradius for high current DD cases. This is shown in Fig. 15 for DD, compared with Fig. 5 for DT, and also in the DD pitch and gyroradius distributions of Figs. 16 and 17. This difference was due to the "delayed loss" component in DD, similar to that described previously $[12,13,16]$, which was significantly reduced or absent in DT. A stronger indication of the absence of delayed loss in DT was seen in I=2.5 MA discharges, as discussed in Sec. 6.2. This difference between DD and DT may be due to the differing collisional loss between $1 \mathrm{MeV}$ tritons and $3.5 \mathrm{MeV}$ alphas.

The total neutron-normalized DD fusion product loss vs. plasma current is shown in Fig. 18, analyzed similarly the DT data in Fig. 10. The loss during DD decreases about as expected from the first-orbit loss model, as it did for the DT cases. The signal levels per neutron for DT were $\approx 1.5$ times larger than those for DD, which was roughly consistent with the expected scintillator response to the respective fusion products (see Sec. 4.2).

Not shown explicitly in Fig. 18 are the DD discharges with MHDinduced fusion product loss, such as that illustrated in Fig. 15, where there was a sudden factor-of-two increase in the DD fusion product loss coincident with a minor disruption at $3.45 \mathrm{sec}$. This MHD-induced loss is characterized by an increase in the loss at low $\rho$ and high $X$, as seen 
previously $[12,16]$. One example of $\mathrm{MHD}$-induced loss in DT is discussed in Sec. 6.3.

\section{Alpha Loss at Other Poloidal Angles}

Alpha loss was also measured for the DT discharges of Table 1 using similar alpha loss detectors at $60^{\circ}$ and $45^{\circ}$ below the outboard midplane. Some of these discharges were also monitored using a similar but movable detector at $20^{\circ}$, with its aperture placed $1.7 \mathrm{~cm}$ radially outside the limiter radius. Here only a brief summary of some typical data from these detectors is presented. A more complete analysis of these signals, which also requires modeling of the $\mathrm{TF}$ ripple loss, will be presented elsewhere.

The alpha loss per neutron at these other three detectors was approximately constant with increasing DT neutron rate at $\mathrm{I}=1.8 \mathrm{MA}$, as it was for the $90^{\circ}$ detector data shown in Fig. 9. This is significant, since the neutral beam losses due to TAE modes in DIII-D were concentrated near the outboard midplane [20], and since the TAE-induced alpha losses seen in numerical simulations were also localized just below the outboard midplane [21]. The absence of any significant increase in alpha loss in the $45^{\circ}$ and $20^{\circ}$ detectors reinforces the conclusion that there were no "collective" alpha loss processes in the high-powered DT experiments.

The alpha loss rate per neutron at these other three detectors was also generally constant vs. time, similar to the $90^{\circ}$ data shown in Figs. 7 and 8. However, in some discharges there was a gradual increase in the loss rate at the $20^{\circ}$ detector, and to a lesser extent in the $45^{\circ}$ detector, over the whole duration of the NBI pulse. The mechanism for this slow increase in the normalized alpha loss at $20^{\circ}$ and $45^{\circ}$ is not yet understood, but might be associated with a diffusive component of TF ripple loss [22]. Note that this is not the same as the "delayed loss" seen previously [12], since that loss was seen only in the $90^{\circ}$ detector, and was not seen in DT.

The normalized alpha loss vs. plasma current in the $60^{\circ}$ detector behaved like that at $90^{\circ}$, in that it generally decreased with plasma current from I=0.6 MA to 2.5 MA similarly to the predictions of the first-orbit model. However, alpha loss at the $45^{\circ}$ detector decreased by $\approx 30 \%$ between $I=1.8 \mathrm{MA}$ and $\mathrm{I}=0.6 \mathrm{MA}$, instead of increasing by a factor of three as predicted by the Lorentz orbit code [23]. Since the DD data shows the same trend vs. plasma current as the DT data, this was evidently a "single particle" loss process common to DD and DT and not a new "collective" alpha effect. A somewhat similar behavior was seen for the DT alpha collection fraction in the $20^{\circ}$ detector, which decreased from $\mathrm{I}=1.0 \mathrm{MA}$ to $\mathrm{I}=0.6 \mathrm{MA}$, similar to previous $\mathrm{DD}$ results obtained with the $20^{\circ}$ probe $[15,22]$. Some of these results may be due to the effects of $T F$ ripple loss, which are expected to be larger toward the outboard midplane (see Appendix II). 


\section{Summary and Discussion}

This paper described measurements and analysis of DT alpha particle loss in TFTR, mainly concentrating on the detector located at the vessel bottom in the ion grad-B drift direction. In general, the DT alpha particle loss processes were similar to those previously seen for DD fusion products. No new "collective" alpha particle loss processes have yet been observed. A discussion in terms of the various alpha loss mechanisms is given below.

\subsection{First-Orbit Loss}

The alpha loss data for the $90^{\circ}$ detector was entirely consistent with the simple first-orbit loss model. For example, the alpha collection fraction at fixed plasma current was independent of the DT neutron rate, as expected for a "single particle" loss mechanism, and the pitch angle and gyroradius distributions agreed well with the calculated first-orbit loss. The plasma current dependence of the alpha loss was also consistent with the first-orbit loss model for the $90^{\circ}$ detector. The alpha loss in the $45^{\circ}$ and $20^{\circ}$ detectors was not consistent with the first-orbit loss model alone. This behavior may be due to the additional effects of $\mathrm{TF}$ ripple-induced alpha loss.

\subsection{Delayed Loss}

The "delayed loss" which was routinely seen in the $90^{\circ}$ detector at high current in DD was much reduced or absent for DT alphas. This was illustrated in Sec. 4.9 for plasmas with $\mathrm{I}=2.0 \mathrm{MA}$, which had a relatively small delayed loss in DD. A clearer example of the absence of delayed loss in DT is shown in Fig. 19 for I=2.5 MA plasmas, for which the delayed loss in DD was relatively larger [12]. This absence of delayed loss also occurred for the (few) $R=2.45 \mathrm{~m}$ plasmas made so far in DT, which tended to have a larger delayed loss in DD than discharges at $R=2.52 \mathrm{~m}$ [16].

The absence of delayed loss in DT suggests that it is caused by a collisional "single particle" effect, since the collisionality changes significantly between DD tritons and DT alphas, whereas the gyroradiusdependent first-orbit loss and collisionless TF ripple loss does not. For example, the ratio of the pitch angle scattering time to the energy e-folding time for $1 \mathrm{MeV}$ tritons is only $\approx 2$, whereas for alpha particles it is $\approx 15$ [16], i.e. tritons suffer more pitch angle scattering than alphas, and so presumably have more collisional loss (this ratio is $\approx 25$ for $3 \mathrm{MeV}$ protons).

The theory of classical collisional pitch angle scattering into the firstorbit loss cone has recently been developed and applied to TFTR [24]. However, quantitative predictions have not yet been made for the collisional loss fraction to the local $90^{\circ}$ detector for these DT vs. DD fusion products. 


\subsection{TF Ripple Loss}

Previous study of DD fusion product loss to the $20^{\circ}$ detector showed a fairly good agreement between the measurements and the collisionless stochastic TF ripple diffusion model incorporated into the MAPLOS code $[15,22]$. A comparable study of the $20^{\circ}$ detector signals for DT alphas has not yet been made. Modeling of TF ripple loss also needs to be improved to incorporate collisional effects [25]. Since alpha ripple loss is an important consideration for the design of the first wall of ITER [1], specific DT experiments on alpha ripple loss are also planned.

\subsection{MHD-Induced Loss}

There was no significant time-averaged increase in the alpha loss associated with background plasma MHD activity in the DT discharges analyzed for this paper (i.e. those in Table 1). Based on the scatter and uncertainties in the data shown in Figs. 9 and 10, an upper limit to potential MHD-induced loss at I $\geq 1.8 \mathrm{MA}$ in this data set is roughly $\approx 50 \%$ of the first-orbit loss level. In DD, large coherent MHD activity was observed to increase the fusion product loss by up to a factor of 3 at I $\geq 1.6 \mathrm{MA}[13,16]$. It should be noted, however, that it is now more difficult to observe the fluctuations in the DT (or DD) fusion product loss than it was in prior years, since the signal-to-background level was reduced by the change in the scintillator material for the DT run (see Sec. 2).

The apparently low level of MHD-induced alpha loss in this data was partially due to the intentionally low level of MHD activity created in many of these DT discharges, such as those in the moderate-power plasma current scan at I=0.6-1.8 MA, and in the modest-power alpha heating experiment at $\mathrm{I}=1.8 \mathrm{MA}$. In general, the level of plasma MHD activity was similar in DD and DT [26], and in a broader database of DT shots there have been many examples of transient MHD-induced alpha loss during sawteeth, fishbones, coherent modes and minor disruptions (to be described elsewhere). These MHD-induced alpha losses appear to be at least qualitatively similar to behavior previously seen in $\mathrm{DD}$.

By far the largest MHD-induced loss yet observed during DT has been during major disruptions, as illustrated in Fig. 20. In this discharge the alpha loss increased by more than a factor of 100 during the period of large $\mathrm{MHD}$ activity $\approx 2 \mathrm{msec}$ just before the plasma current started to decay. The complicated time dependence of the alpha loss during this disruption is probably due to the kink and ballooning modes observed during this time [26]. Similar behavior was seen previously during disruptions in DD [16], so there is no reason to believe that this DT loss is a collective alpha effect. It is interesting that the relative increase in the alpha loss at the $90^{\circ}$ detector in this disruption was much larger than that in the $60^{\circ}$ or $45^{\circ}$ detectors, as if the alpha loss during disruption was mainly in the vertical 
direction. Such an alpha loss mechanism may be a concern for ITER, since apparently $\approx 10 \%$ of the confined alphas were lost during this time.

\subsection{Collective Alpha Effects}

No alpha particle loss due to any new "collective" alpha instabilities was observed in the DT experiments analyzed in this paper [5]. This is not surprising, since only one of these discharges (\#76770) showed a slight increase in activity at the Alfven-frequency which might have been associated with TAE modes [26]. However, even this discharge, which had the highest sustained neutron rate of 7.5 MW, did not show any significant increase in alpha loss with respect to other discharges at that plasma current, as shown in Fig. 9 (\#767.70 is the shot with the highest sustained neutron rate at 2.5 MA). This was probably because the level of the Alfven activity in this discharge was still much smaller than the Alfven activity observed during fast ion loss during NBI or ICRH minority ion heating [26].

\subsection{Conclusions}

Alpha particle loss was measured during the first year of TFTR DT run with a detector $90^{\circ}$ below the outer midplane in the ion grad-B drift direction. The alpha loss seen in this detector during DT was dominated by the classical "single-particle" first-orbit loss process. No signs of any new "collective" alpha particle loss processes were seen up to the maximum sustained fusion power level of $10.7 \mathrm{MW}$, i.e. the alpha collection fraction at a constant plasma current was independent of the DT neutron rate up to an alpha source power level of $\approx 2 \mathrm{MW}$. This was also true for the alpha signals seen in the other detectors (described only briefly here).

In addition to the absence of any collective alpha loss process, these initial DT results also appear to show a relatively lower level of MHDinduced loss and "delayed loss" than the corresponding DD discharges $[13,16]$. One potential explanation for this could be based on the different single-particle collisionality of $3.5 \mathrm{MeV}$ alphas and the $1 \mathrm{MeV}$ triton fusion product. The DD tritons have a $\geq 3$ times longer slowing-down time than DT alphas, so that the confined triton population susceptible to MHD or delayed loss should be larger than that for alphas, relative to their comparable firstorbit loss fractions. The tritons also have a larger pitch-angle scattering over their thermalization time, potentially leading to increased collisional loss [24].

Further experimental work is needed in several areas before drawing any final conclusions concerning alpha particle loss in TFTR DT. In particular, more analysis is needed on the alpha loss to the detectors at $60^{\circ}, 45^{\circ}$ and $20^{\circ}$ below the outer midplane, along with improved calculations of the expected collisional TF ripple loss. The MHD-induced alpha loss effects also need to be examined and modeled more carefully. 
Acknowledgements: We thank C.W. Barnes, M. Bell, C.E. Bush, R.L. Boivin, Z. Chang, C.Z. Cheng, M. Diesso, H. Duong, J. Felt, G.Y. Fu, H. P. Furth, G. LeMunyan, R.J. Hawrykuk, W.W. Heidbrink, D.W. Johnson, K. McGuire, D.M. Meade, and D.A. Spong, M. Tuszewski for their various contributions and support for this work, which was performed under DOE Contract \#DE-AC02-76CH0-3073. 


\section{References}

1) S. Putvinski, et al, Proc. 15th Int'l. Conf. on Plasma Physics and Cont. Nucl. Fusion Research, Seville, Spain, IAEA '94

2) W. W. Heidbrink and G. Sadler, Nuclear Fusion 34, 535 (1994)

3) C.Z. Cheng, et al, Proc. 14th Int'l. Conf. on Plasma Physics and Cont. Nucl. Fusion Research, Wurzburg, 1992, IAEA-CN-56/ D-2-1

4) S.J. Zweben, et al, Nucl. Fusion 28(12), 2230 (1988)

5) J.D. Strachan, et al, Phys. Rev. Lett. 72(22), 3526 (1994)

6) R.J. Hawryluk, et al, Phys. Rev. Lett. 72(22), 3530 (1994)

7) R. Boivin, et al, Rev. Sci. Inst. 63, 4418 (1992)

8) S. J. Zweben, Rev. Sci. Inst. 63, 4565 (1992)

9) M. Tuszewski and S. J. Zweben, Rev. Sci. Inst. 64(9), 2459 (1993)

10) D. S. Darrow, et al, "Measurements of Loss of DT Fusion Products Using Scintillator Detectors in TFTR", to be pub. in Rev. Sci. Inst.

11) S.J. Zweben, et al, Nucl. Fusion 31, 2219 (1991)

12) S.J. Zweben, et al, Nucl. Fusion 33, 705 (1993)

13) S.J. Zweben, et al, Proc. 14th Int'l. Conf. on Plasma Physics and Cont. Nucl. Fusion Research, Wurzburg, 1992, paper IAEA-CN-56/A-6-3.

14) S.J. Zweben,R.L. Boivin, M Diesso, et al, Nuclear Fusion 30, 1551 (1990)

15) R. L Boivin, S.J. Zweben, and R.B. White, Nucl. Fusion 33, 449 (1993)

16) S. Zweben, et al, Phys. Plasmas 1(5), 1469 (1994)

17) J. Felt, et al, Rev. Sci. Inst. 61(10) 3262 (1990)

18) H. G. Adler, et al, to be published in Rev. Sci. Inst. (1994); A.T. Ramsey, PPPL-3010 (1994)

19) L.C. Johnson, et al, to be published in Rev. Sci. Inst. (1994)

20) H.H. Duong, W.W. Heidbrink, E.J. Strait, R.L. Lee, R. Moyer, T.W. Petrie, and J. Watkins, Nucl. Fusion 33(5) 1993, pg.749.

21) D.J. Sigmar, C.T. Hsu, R.B. White, C.Z. Cheng, Phys. Fluids B 4, 1506 (1992)

22) R.L. Boivin and S.J. Zweben, Phys. Fluids B 5, 1559 (1993)

23) S.J. Zweben, Proc. 21st EPS Conference, Montpellier 1994, paper A27

24) C.S. Chang, et al, Princeton Plasma Physics Laboratory Report PPPL3005 (1994), to be published in Phys. Plasmas

25) M.H. Redi, et al Princeton Plasma Physics Lab Report PPPL-3011 (1994)

26) E.D. Fredrickson, et al, Proc. 15th Int'l. Conf. on Plasma Physics and Cont. Nucl. Fusion Research, Seville, Spain, IAEA '94

27) R.B. White and H.E. Mynick, Phys. Fluids B1, 980 (1989)

28) R.V. Budny, to be published in Nucl. Fusion (1994)

29) L.M. Hively and G.H. Miley, Nucl. Fusion 20, 969 (1980)

30) H.E. Mynick, Phys. Fluids B 5(5), 1471 (1993)

31) D. S. Darrow, et al, Princeton Plasma Physics Lab Report PPPL-2975 (1994)

32) L.C. Johnson, PPPL, private communication, 1994

33) F. M. Levinton, Rev. Sci. Instrum. 63, 5157 (1992).

34) S. P. Hirshman, D. K. Lee, F. M. Levinton, S. H. Batha, M. Okabayashi, and R. M. Wieland, Phys. Plasmas 1, 2277 (1994).

35) S. H. Batha, F. M. Levinton, S. P. Hirshman, M. G. Bell, and R. M. Wieland, to be published in Rev. Sci. Instrum (1994). 


\section{Figure Captions}

1) Schematic picture of the design of the TFTR lost alpha detector, which was located $90^{\circ}$ below the outboard midplane. The escaping alphas enter a pair of apertures which disperse them in pitch angle and gyroradius. The 2 -D image of the visible light emission from the scintillation screen is transmitted to a gated intensified video camera for analysis.

2) Calculated loss orbits for $3.5 \mathrm{MeV}$ alpha particles to the $90^{\circ}$ detector in TFTR for plasma currents of $I=0.6$ and 2.0 MA. At $I=0.6 \mathrm{MA}$ this detector "sees" first-orbit alpha loss from the plasma center, while at I=2.0 MA it does not, which results in a lower alpha loss rate at the higher current. The pitch angle of the maximum alpha loss is $X \approx 78^{\circ}$ at $I=0.6 \mathrm{MA}$, but $\chi \approx 60^{\circ}$ at $\mathrm{I}=2.0 \mathrm{MA}$.

3) Raw data of the scintillator light images in the $90^{\circ}$ detector for $\mathrm{I}=0.6 \mathrm{MA}$ and 2.0 MA. The raised square region corresponds to the neutron/gamma background light produced in the quartz fiberoptic bundle under the vessel. The peak corresponds to the alpha loss signal. These images are averaged over 0.4-0.7 sec after the start of NBI for discharges with $a \approx 50 / 50$ DT mix.

4) A map of the $90^{\circ}$ scintillator plane showing the lost alpha data for the $I=2.0 \mathrm{MA}$ shot of Fig. 3, along with the pitch angle $(x)$ vs. gyroradius $(\rho)$ coordinate system used to interpret it. These coordinates are the centroids of the impact positions of ions passing through the entrance apertures at a given $X$ and $\rho$. The pitch angle coordinate runs from $90^{\circ}$ to $45^{\circ}$ from left-toright in $5^{\circ}$ increments, and the gyroradius coordinate runs from $3.5 \mathrm{~cm}$ to 6 $\mathrm{cm}$ in $0.5 \mathrm{~cm}$ intervals, and from 6 to $10 \mathrm{~cm}$ in $1 \mathrm{~cm}$ intervals from bottomto-top. The shaded region is used for neutron background subtraction.

5) Unfolded scintillator images for the data of Fig. 3. The background has been subtracted out, and the light intensity plotted using the pitch angle and gyroradius map shown in Fig. 4. The contours are drawn in steps of $10 \%$ of the maximum signal level.

6) Time dependences of the net alpha loss signals for comparable DT and $\mathrm{DD}$ discharges, both at $\mathrm{I}=2.0 \mathrm{MA}$ and $26 \mathrm{MW}$ of NBI over 3-4 sec. These signals were integrated over the $(X, \rho)$ map of Fig. 4 , and the neutron background was subtracted out. The DD signals are $\leq 5 \%$ of those during DT, and so are negligible in the analysis of the DT discharges.

7) Time dependence of the scintillator lost alpha signals and neutron background levels for DT discharges at I=0.6 MA, 1.8 MA, and 2.0 MA. These signals are normalized to each other at their peak values. The neutron background is due to the fiberoptic fluorescence, which is proportional to the DT neutron rate. The alpha loss signal follows fairly closely the DT neutron signal vs. time. 
8) The time dependence of the neutron-normalized alpha loss for the discharges of Fig. 7. Also shown is the time dependence of the calculated first-orbit alpha loss to the $90^{\circ}$ detector for these discharges. This calculation takes into account the time evolution of the plasma current and neutron source profiles, as modeled by the TRANSP code. The calculated first-orbit loss fraction is roughly independent of time, i.e. similar to the data. The error bars are an estimate of the experimental uncertainty.

9) The alpha particle collection fraction vs. the peak fusion power for discharges at I=2.0 MA, 2.5 MA, and 2.7 MA, integrated over 0.4-0.7 sec after the start of NBI. The alpha collection fraction is the total alpha loss rate into the detector divided by the total neutron rate (normalized as discussed in Sec. 4.4). The alpha collection fractions are roughly independent of the DT neutron rate at a given current, showing the absence of any significant "collective" effect at the high fusion power. The average first-orbit loss rates for each of these currents is also shown; the shot-toshot variations in the calculated first-orbit loss at a given current are typically within $\pm 10 \%$ of this average.

10) Dependence of the alpha collection fraction on the plasma current for the whole set of DT discharges (Table 1). The data is normalized to the calculated first-orbit loss alpha collection fraction at I=0.6 MA, where the first-orbit loss is expected to dominate. All the data fit the first-orbit model within the joint uncertainties, which at $I=2.0 \mathrm{MA}$ are about $\pm 30 \%$ for the modeling and $\pm 30 \%$ for the measurements. The relative uncertainties in both the data and modeling decrease toward lower plasma current where the first-orbit loss fraction is largest (see also Appendix 1).

11) Pitch angle distributions of the alpha loss at $I=0.6-1.8 \mathrm{MA}$ for single tritium source discharges. The shapes agrees fairly well with the firstorbit loss calculation made using the TRANSP profiles, which were corrected for the geometric and optical resolution of the detectors. The measured and calculated curves are normalized vertically to each other near their peaks, but the horizontal axis was absolutely calibrated by an invessel alignment to within $\approx 3^{\circ}$.

12) Pitch angle distributions of alpha loss for two DT discharges at $I=2.0$ MA, one with a single tritium source (\#73234) and the other with full DT (\#73268). There is no significant difference between the pitch angle distributions for these two cases, indicating the absence of any new alpha loss process at high power in DT, and both distributions agree well with the calculated shape of the first-orbit loss distribution. The measured and calculated curves are normalized vertically to each other near their peaks, but there the horizontal axis was absolutely calibrated by an in-vessel alignment to within $\approx 3^{\text {? }}$.

13) Gyroradius distributions of alpha loss at $I=0.6-1.8 \mathrm{MA}$ for single tritium source discharges. In all cases the shapes agrees fairly well with the model calculations for first-orbit alpha loss at $3.5 \mathrm{MeV}$ alphas, after 
correcting for the geometric and optical resolution of the detectors. The measured and calculated curves are normalized vertically to each other near their peaks, but there the horizontal axis was absolutely calibrated by an in-vessel alignment to within $\approx 1 \mathrm{~cm}$ on this scale.

14) Gyroradius distributions of alpha loss for two discharges at $I=2.0 \mathrm{MA}$, one with a single tritium source (\#73234) and the other a full DT discharge (\#73268). There is no significant difference in the measured distributions for these two cases. Model calculations are shown for three different assumed alpha particle energies, the best agreement being with the 3.5 $\mathrm{MeV}$ model curve, as expected for first-orbit alpha loss. Note that the toroidal field was $\approx 5 \%$ higher than for the discharges in Fig. 13, causing a slight decrease in the gyroradius. The measured and calculated curves are normalized vertically to each other near their peaks, but there the horizontal axis was absolutely calibrated by an in-vessel alignment to within $\approx 1 \mathrm{~cm}$ on this scale.

15) Pitch angle vs. gyroradius distributions for $D D$ fusion product loss at $I=$ 0.6 and 2.0 MA. These distributions are similar to those for similar DT discharges (as shown in Fig. 5), except for the presence of a small anomalous "delayed" loss component at high pitch angles $\left(X \approx 70^{\circ}\right)$ and low gyroradius $(p \approx 4 \mathrm{~cm})$ at $I=2.0 \mathrm{MA}$, which was not present in the comparable DT shot. The strength of this anomalous delayed loss feature in DD can increase substantially during MHD activity, as shown in the bottom case.

16) Pitch angle distributions for $\mathrm{DD}$ fusion product loss at $\mathrm{I}=0.6 \mathrm{MA}$ and 2.0 MA. The shape of the measured distributions were similar to that for the DT discharges of Figs. 14 and 15, as expected from the first-orbit loss model, except for the presence of a small delayed loss component in the data at $x=70^{\circ}$ in $\mathrm{DD}$.

17) Gyroradius distributions for $\mathrm{DD}$ fusion product loss at $\mathrm{I}=0.6 \mathrm{MA}$ and 2.0 MA. The distributions for DD fusion products were peaked at a slightly smaller gyroradius than those for DT, as expected from the first-orbit loss model. The discharges at I $=2.0 \mathrm{MA}$ also had $\mathrm{a} \approx 5 \%$ smaller toroidal field which also changed the gyroradius by about this fraction about as expected.

18) Dependence of the neutron-normalized DD fusion product loss on plasma current, analogous to the DT data in Fig. 10. The data is normalized to the calculated first-orbit alpha collection fraction at $\mathrm{I}=0.6 \mathrm{MA}$ where first-orbit loss is expected to dominate. The data fit the first-orbit model for $\mathrm{I} \leq 1.8 \mathrm{MA}$, but the presence of an additional delayed loss component can be seen at I $\geq 2.0 \mathrm{MA}$. These data points represent all the DD shots in Table 1.

19) Comparison between the pitch angle vs. gyroradius distributions of $D D$ and $\mathrm{DT}$ discharges at $\mathrm{I}=2.5 \mathrm{MA}$. The delayed loss component in $\mathrm{DD}$ at $\mathrm{X} \approx 65$ $70^{\circ}$ pitch angle and $\rho \approx 3-4 \mathrm{~cm}$ gyroradius is not seen in DT. The most likely 
cause is the difference in classical pitch angle scattering between $1 \mathrm{MeV}$ tritons in $\mathrm{DD}$ and $3.5 \mathrm{MeV}$ alphas in DT.

20) A discharge in which there was a large increase in DT alpha particle loss just prior to a major disruption, with $\mathrm{I}=2.5 \mathrm{MA}, 33 \mathrm{MW}$ of NBI, and 9.2 MW of fusion power (\#76778). The plasma current has not changed during this time, but large MHD activity was observed. Smaller increases in alpha loss were seen in the other detectors. Similar disruption-induced fusion product loss was seen in DD discharges.

21) Calculations of the global (i.e. total) alpha particle loss in TFTR vs. plasma current from various codes for some of the discharges of Table 1. The first-orbit loss calculations agree reasonably well with each other, the main differences being due to slightly different modeling of the discharge profiles and wall. The calculated $\mathrm{TF}$ ripple loss increases substantially between collisionless and collisional models, but the collisional axisymmetric losses are small, according to TRANSP.

22) Calculations of the shape of the poloidal distribution of $3.5 \mathrm{MeV}$ alpha loss in TFTR based on the MAPLOS code. The shaded regions represent smoothed approximations to the Monte Carlo results (dotted lines). For both $\mathrm{I}=0.6$ and $1.8 \mathrm{MA}$ the first-orbit loss has a broad peak along the vessel bottom between $60^{\circ}$ and $90^{\circ}$ below the outboard midplane. At $\mathrm{I}=1.8 \mathrm{MA}$ there is a significant TF ripple-induced alpha loss component at $\leq 30^{\circ}$ below the outboard midplane. The TF ripple loss calculated using the collisional ORBIT code model also predicts alpha loss to be localized $\leq 30^{\circ}$ below the outboard midplane.

23) Comparison of the TRANSP calculations of the DT neutron source profile with measurements from the vertical neutron collimator for a highpower DT discharge at I=1.8 MA (\#73446). In both cases the neutron emission is integrated over a vertical chord corresponding to the detector location. The small differences between the two profiles result in a $\approx 15 \%$ change in the calculated first-orbit loss to the $90^{\circ}$ detector for this discharge.

24) The $q(R)$ profiles at 0.6 seconds after the start of NBI computed by VMEC from the MSE measurements (solid line), and by TRANSP (dashed line) for single tritium source shots at $\mathrm{I}=0.6-1.8 \mathrm{MA}$.

25) The first-orbit alpha collection fraction vs. current computed using either the symmetrized MSE/VMEC $q(r)$ profile (solid line) or the TRANSP $q(r)$ profile (dashed line). The difference between the two is less than $10 \%$. 
Table 1 - Shot List (DT Shots in Boldface, $\approx 2.5 \mathrm{MW}$ for each beam source)

\begin{tabular}{|c|c|c|c|c|}
\hline shot \# & $\begin{array}{l}\text { Current } \\
\text { (MA) }\end{array}$ & $\begin{array}{l}\text { NBI Power } \\
(\mathrm{MW})\end{array}$ & $\begin{array}{l}\text { Bcams } \\
\# \mathrm{~T} / \# \mathrm{D}\end{array}$ & $\begin{array}{c}\text { Neutron rate } \\
(\times 10 \quad 17 / \mathrm{sec}) \\
\end{array}$ \\
\hline 73300 & 0.6 & 5 & $1 / 1$ & 0.45 \\
\hline 73301 & 0.6 & 5 & $1 / 1$ & 0.48 \\
\hline 73302 & 0.6 & 5 & $1 / 1$ & 0.48 \\
\hline 72988 & 0.6 & 5 & $0 / 2$ & 0.01 \\
\hline 72989 & 0.6 & 5 & $0 / 2$ & 0.01 \\
\hline 73311 & & & 71 & \\
\hline & 1.0 & 10 & $1 / 3$ & 1.90 \\
\hline 73312 & 1.0 & 10 & $1 / 3$ & 1.80 \\
\hline 72993 & 1.0 & 10 & $0 / 4$ & 0.05 \\
\hline 73314 & 1.4 & 10 & $1 / 3$ & 2.38 \\
\hline 73315 & 1.4 & 10 & $1 / 3$ & 2.40 \\
\hline 72994 & 1.4 & 10 & $0 / 4$ & 0.06 \\
\hline 73306 & 1.8 & 13 & $1 / 4$ & 3.13 \\
\hline 73307 & 1.8 & 13 & $1 / 4$ & 3.27 \\
\hline 73446 & 1.8 & 22 & $5 / 3$ & 13.10 \\
\hline 73447 & 1.8 & 21 & $7 / 0$ & 7.86 \\
\hline 73450 & 1.8 & 23 & $8 / 0$ & 9.55 \\
\hline 73452 & 1.8 & 20 & $5 / 2$ & 11.00 \\
\hline 73457 & 1.8 & 20 & $2 / 6$ & 7.83 \\
\hline 72990 & 1.8 & 10 & $0 / 4$ & 0.03 \\
\hline 72991 & 1.8 & 15 & $0 / 6$ & 0.08 \\
\hline 73222 & 2.0 & 13 & $1 / 4$ & 3.00 \\
\hline 73228 & 2.0 & 8 & $1 / 2$ & 2.20 \\
\hline 73229 & 2.0 & 6 & $1 / 1$ & 1.20 \\
\hline 73234 & 2.0 & 28 & $1 / 10$ & 6.80 \\
\hline 73235 & 2.0 & 24 & $4 / 5$ & 14.30 \\
\hline 73254 & 2.0 & 27 & $4 / 6$ & 14.90 \\
\hline 73258 & 2.0 & 27 & $5 / 5$ & 16.10 \\
\hline 73268 & 2.0 & 29 & $7 / 4$ & 20.30 \\
\hline 73273 & 2.0 & 22 & $7.5 / 0$ & 7.90 \\
\hline 72635 & 2.0 & 19 & $8 / 0^{\circ}$ & 0.76 \\
\hline 72695 & 2.0 & 20 & $5.5 / 3^{*}$ & 0.51 \\
\hline 73219 & 2.0 & 13 & $0 / 5$ & 0.10 \\
\hline 73220 & 2.0 & 13 & $0 / 5$ & 0.10 \\
\hline 76746 & 2.5 & 24 & $4 / 4$ & 15.00 \\
\hline 76747 & 2.5 & 27 & $5 / 4$ & 20.00 \\
\hline 76748 & 2.5 & 30 & $6 / 4$ & 22.50 \\
\hline 76769 & 2.5 & 28 & $6 / 3$ & 21.20 \\
\hline 76770 & 2.5 & 33 & $6 / 5$ & 24.00 \\
\hline 76771 & 2.5 & 31 & $6 / 4$ & 23.20 \\
\hline 76773 & 2.5 & 30 & $6 / 4$ & 19.80 \\
\hline 76743 & 2.5 & 21 & $0 / 8$ & 0.46 \\
\hline 76744 & 2.5 & 25 & $0 / 9$ & 0.54 \\
\hline 76745 & 2.5 & 28 & $0 / 10$ & 0.55 \\
\hline
\end{tabular}


Table 1 - Shot List ...cont...

\begin{tabular}{|l|l|l|l|l|}
\hline shot \# & $\begin{array}{l}\text { Current } \\
(\mathrm{MA})\end{array}$ & $\begin{array}{l}\text { NBI Power } \\
(\mathrm{MW})\end{array}$ & $\begin{array}{l}\text { Beams } \\
\text { \#T/\#D }\end{array}$ & $\begin{array}{l}\text { Neutron rate } \\
(\mathrm{x} 1017 / \mathrm{sec})\end{array}$ \\
\hline
\end{tabular}

\begin{tabular}{|l|l|l|l|l|}
\hline & & & & \\
\hline 80506 & 2.7 & 36 & $7 / 5$ & 27.5 \\
\hline 80507 & 2.7 & 31 & $7 / 5$ & 21.2 \\
\hline 80537 & 2.7 & 36 & $7 / 5$ & 24.0 \\
\hline 80539 & 2.7 & 39 & $7 / 5$ & $36.4 \star \star$ \\
\hline 80541 & 2.7 & 32 & $7 / 5$ & 23.6 \\
\hline 80542 & 2.7 & 40 & $7 / 5$ & 24.6 \\
\hline 80543 & 2.7 & 32 & $7 / 5$ & 20.5 \\
\hline 80544 & 2.7 & 36 & $7 / 5$ & 26.3 \\
\hline
\end{tabular}

* $\mathrm{T}$ beams contain $2 \%$ tritium \& $98 \%$ deuterium (i.e. trace tritium) ** alpha loss evaluated during 3.7-3.75 sec near the neutron peak 
Table 2: Alpha Particle Parameters Calculated by TRANSP for Shot \#76770

$\begin{array}{ll}\text { Major Radius } & 2.52 \mathrm{~m} \\ \text { Minor Radius } & 0.87 \mathrm{~m} \\ \text { Plasma Current } & 2.5 \mathrm{MA} \\ \text { NBI Power } & 33 \mathrm{MW} \\ \text { Fusion Power } & 7.5 \mathrm{MW} \\ \text { Alpha Power } & 1.5 \mathrm{MW} \\ <\beta> & 1.01 \% \\ <\beta \alpha> & 0.03 \% \\ \beta \alpha(0) & 0.27 \% \\ \beta(0) & 3 \% \\ \mathrm{R} \nabla \beta \alpha & 0.02 \\ \mathrm{~W}_{\text {tot }} & 6.2 \mathrm{MJ} \\ \mathrm{W} \alpha & 0.2 \mathrm{MJ} \\ \mathrm{V} \alpha / \mathrm{VA}(0) & 1.6 \\ \mathrm{n}_{\alpha}(0) / \mathrm{n}_{\mathrm{e}}(0) & 0.3 \% \\ \text { First-orbit } & \approx 3 \% \\ \text { loss fraction } & \end{array}$




\section{Appendix I: Calculations of Global Alpha Loss .}

The global (i.e. total) alpha loss fractions for some of the TFTR discharges of Table 1 were calculated using several existing codes, with the results shown in Fig. 21. These global loss calculations were not directly used for interpretations of the local alpha loss measurements described in this paper, but are described here for the sake of completeness. The calculations of the expected first-orbit loss into the TFTR alpha loss detectors were made with a Lorentz code (Sec. 3), which takes into account the solid angle of the detector acceptance and the decrease in the angle of the orbit's trajectory toward the wall with increased plasma current. These effects are not taken into account in the calculations described in this Appendix, e.g. only the total alpha loss per unit wall area was calculated.

The MAPLOS code [15] was used to calculate the first-orbit and TF ripple loss using 256,000 ions in a collisionless bounce-averaged orbitfollowing Monte Carlo code in a simplified magnetic geometry. The SNAP code calculated the guiding center orbits of $\approx 2000$ alpha particles born on a poloidal, radial, and pitch angle grid using its model for the plasma current and alpha source profile. Both these models counted alphas hitting the wall in a single orbit as first-orbit loss, and confined trapped alphas whose first banana tips lay in the stochastic TF ripple loss region were counted as TF ripple loss, as in the RIPLOS code [27]. The TRANSP code [28] follows a Monte Carlo distribution of $\approx 6000$ alpha orbits calculated using its version of the alpha source and plasma current profiles, including a model for collisional slowing down and pitch angle scattering, but not TF ripple.

These codes are in general agreement that the first-orbit alpha loss at $\mathrm{I}=0.6 \mathrm{MA}$ is $\approx 40 \pm 20 \%$, while at $\mathrm{I}=2.0 \mathrm{MA}$ it is $\approx 4 \pm 2 \%$. The differences between codes for a given plasma current are partly due to their somewhat different assumptions for the alpha source and plasma current profiles. The TRANSP first-orbit loss results [28] are somewhat higher than those of the other codes, probably because it assumes the vessel wall is closer to the plasma edge (this is modeled more correctly in the other codes).

The TF ripple loss calculated from the collisionless MAPLOS and SNAP codes is $<1 \%$ at I=0.6 MA, since few trapped alphas were confined on their first orbits, and $\approx 5 \%$ from both codes at $\mathrm{I}=2.0 \mathrm{MA}$. However, the collisional TF ripple-induced alpha loss as calculated by the Monte Carlo guiding center code ORBIT was $\approx 20 \%$ [25], i.e. much larger than the collisionless alpha loss. However, these ORBIT calculations were done only for higher-ripple plasmas with $R=2.6 \mathrm{~m}$, and were limited to $\approx 250$ alpha particles.

MAPLOS calculations of the pitch-angle-integrated poloidal distribution of alpha loss for $I=0.6 \mathrm{MA}$ and $\mathrm{I}=2.0 \mathrm{MA}$ cases are shown in Fig. 22. The first-orbit loss was predicted to be broadly distributed in the poloidal direction, whereas the TF ripple loss is predicted to be highly 
localized near the outboard midplane. [15,22]. The pitch-angle-integrated poloidal distribution of TF-ripple induced alpha loss was determined by the collisional ORBIT for one case ( $I=1.8 \mathrm{MA}, \mathrm{R}=2.6 \mathrm{~m})$ was similar to that obtained by MAPLOS, i.e. the TF-ripple induced alpha loss is localized to within $\leq 30^{\circ}$ of the outboard midplane [25]. Therefore TF ripple-induced alpha loss should not be visible in the $90^{\circ}$ lost alpha data analyzed in this paper.

Several other fusion product loss mechanisms have been investigated recently. Calculations of axisymmetric collisional loss due to pitch angle scattering of alphas born near the passing/trapped boundary into the firstorbit loss cone have been made using a simplified analytic model, and compared with the Monte Carlo calculations in TRANSP [24]. The calculated poloidal distribution of collisional loss (without TF ripple) was similar to that of first-orbit loss, i.e. peaked near the bottom of the vessel, but the magnitude of this loss was much less than first-orbit loss (Fig. 21). This is consistent with earlier calculations of non-prompt alpha loss [29].

The effect of helical magnetic perturbations due to internal plasma MHD activity on alpha loss has been calculated previously using the GC3 code $[16,30]$. However, in the DT experiments described in this paper there was no observable MHD-induced increase in the alpha loss, except during disruptions (Sec. 6.3). ICRH-induced loss has been observed and modeled in DD plasmas [31], but the present experiments did not have any ICRH.

\section{Appendix II: Comparison of TRANSP Profiles with Measurements}

The calculations of first-orbit alpha loss in Sec. 3 used as input the neutron source profiles $S(r)$ and the $q(r)$ profiles calculated by the timedependent TRANSP transport code [28], which were available for almost all of the discharges discussed in this paper. This Appendix gives some typical comparisons between the TRANSP calculations and measurements of these quantities which were available for some of the discharges discussed in this paper.

A comparison between the measured $S(r)$ profile and the TRANSP modeling of this profile is shown in Fig. 23 for one of the I=1.8 MA high powered DT discharges (\#73346). For this comparison the TRANSP profile was integrated over 9 vertical chords for a direct comparison with the sightlines of the vertical multichannel neutron collimator [32]. The agreement is fairly good for both the profile shape and for the absolute neutron emission level.

The Abel-inverted neutron source profiles from the neutron collimator data were calculated and read into the Lorentz orbit code, and the alpha collection fraction was calculated in the same way as for the TRANSP neutron profiles, while keeping the magnetic configuration 
constant. The resulting first-orbit alpha collection fraction for the $90^{\circ}$ detector was $\approx 15 \%$ higher than for the TRANSP neutron source profile for this case, which is within the estimated $\approx 30 \%$ uncertainty in the calculation of this loss. Similar variations in the neutron source profiles at lower plasma current would result in a smaller change in resulting alpha collection fraction, as discussed in Sec. 3.

The calculations of alpha collection fractions also depended on the TRANSP-calculated $\mathrm{q}(\mathrm{r})$ profiles. A consistency check of TRANSP was made using data from the motional-Stark-effect (MSE) diagnostic [33]. The MSE diagnostic measures the internal magnetic field pitch angle profile at up to 12 locations in the midplane of the tokamak. These data, along with external magnetic field and internal kinetic profile information (including fast particle effects) was used by the free-boundary equilibrium code VMEC [34] to find a self-consistent magnetohydrodynamic equilibrium.

The $q(R)$ profiles computed by VMEC and by TRANSP are shown in Fig. 24 for the discharges at four different plasma currents (from Table 1). For plasma currents greater than $\mathrm{I}=0.6 \mathrm{MA}$, the two calculations differed by less than $20 \%$. At the lowest current of $\mathrm{I}=0.6 \mathrm{MA}$, the discrepancy was somewhat larger on the outside of the plasma. From Monte Carlo analysis of the entire MSE/VMEC data analysis procedure, it was estimated that the uncertainty in $\mathrm{q}(0)$ was $7 \%$, while the uncertainty in the rest of the profile was less than $10 \%$ [35]. A similar error analysis of the TRANSP q profile has not yet been performed.

The Lorentz orbit code was used to calculate the first-orbit alpha collection fraction using both the MSE/VMEC ad TRANSP $q(r)$ profiles, which were derived by symmetrizing the $q(R)$ profiles, while keeping the neutron source profile fixed. As shown in Fig. 25, the results for all currents were within $\approx 10 \%$ of each other, which was well within the estimated $\approx 30 \%$ uncertainty in this calculation. Thus the TRANSPcalculated $q$ profiles were sufficiently accurate for calculations of the alpha collection fraction. Such good agreement between VMEC and TRANSP was not typical. The agreement in these discharges occurs because these cases do not have significant MHD activity, sawteeth, or non-inductive current, all of which can cause substantial deviations from the TRANSP results. 


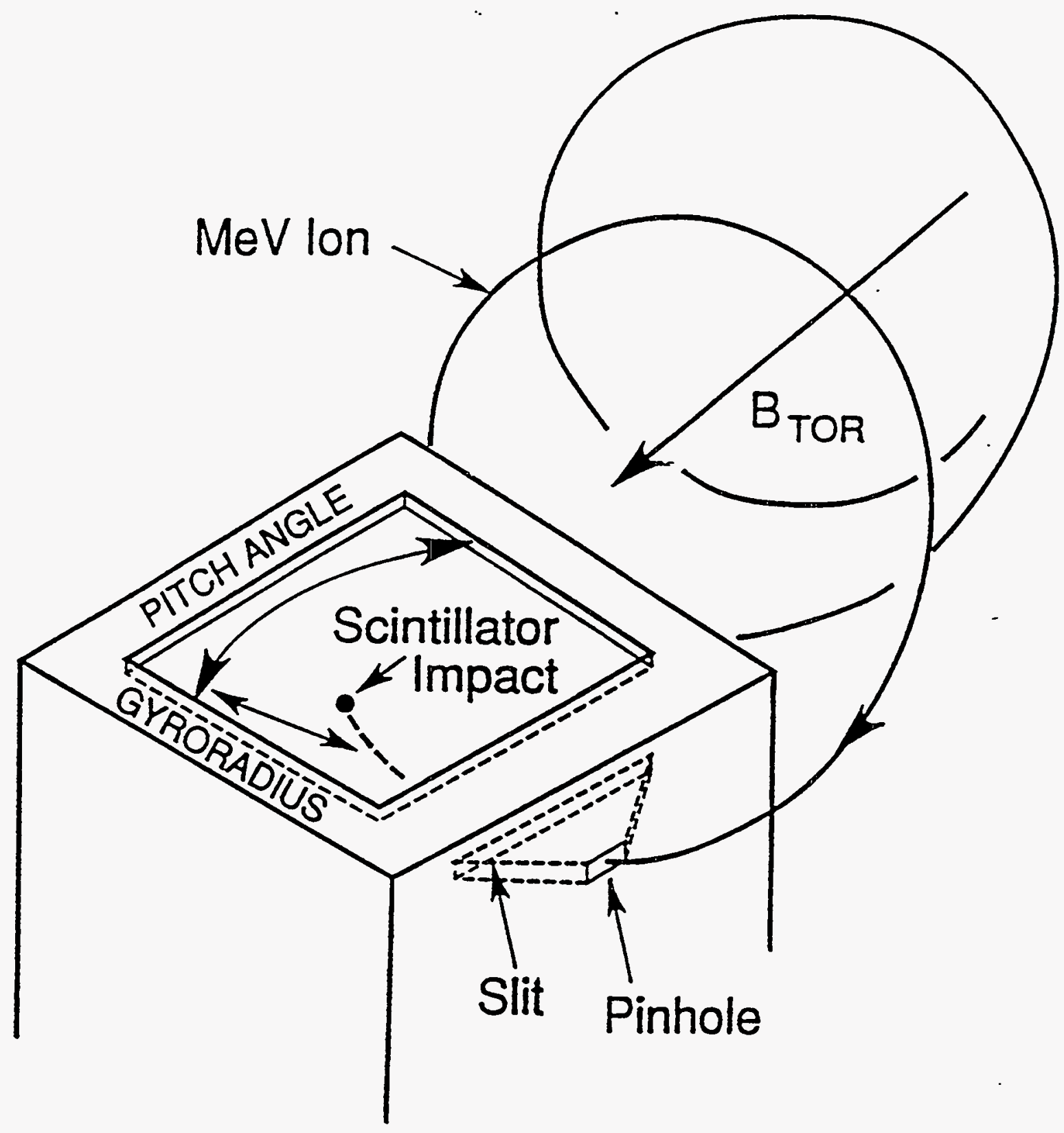

Fig. 1 

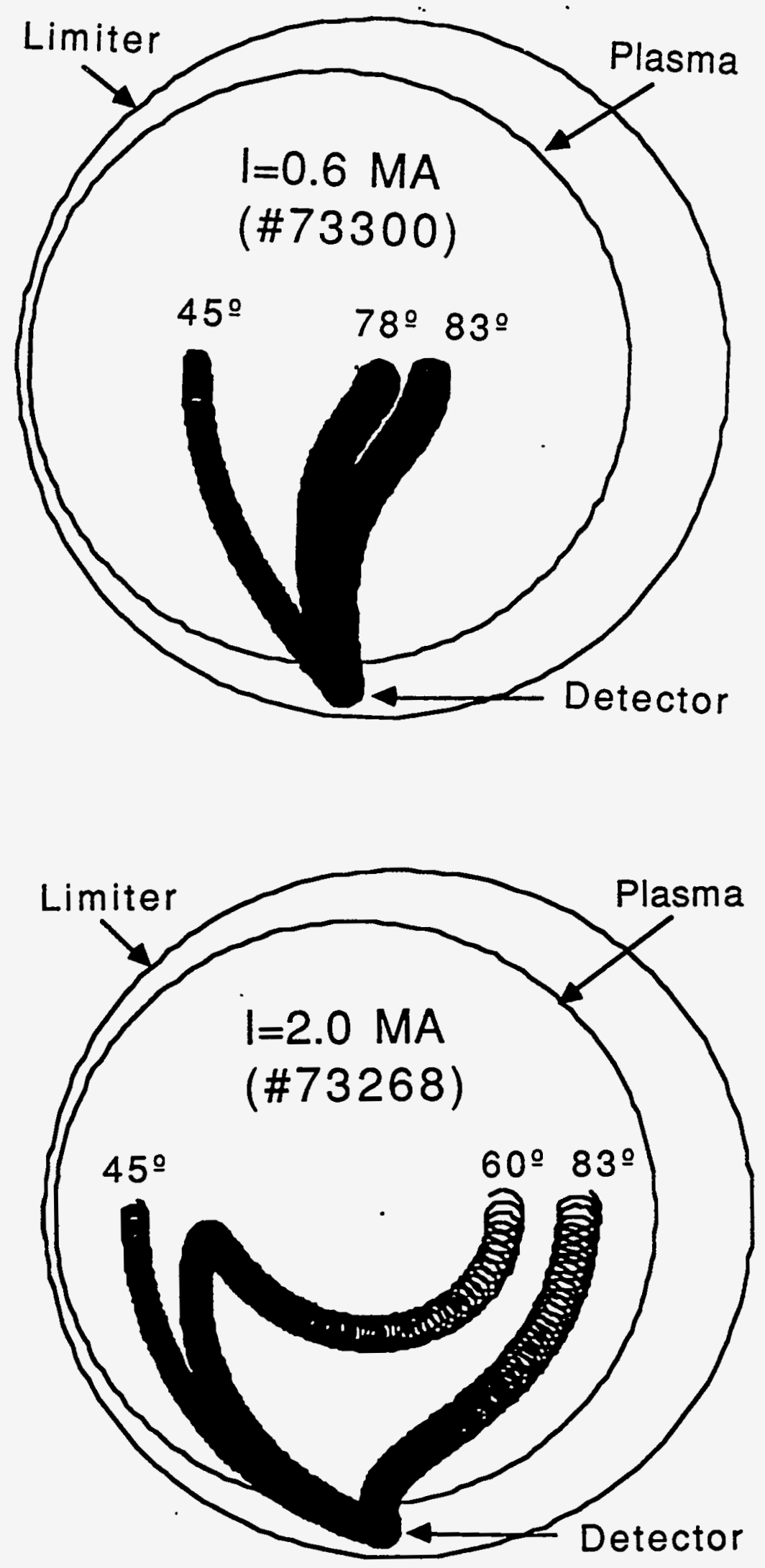

Fig. 2 

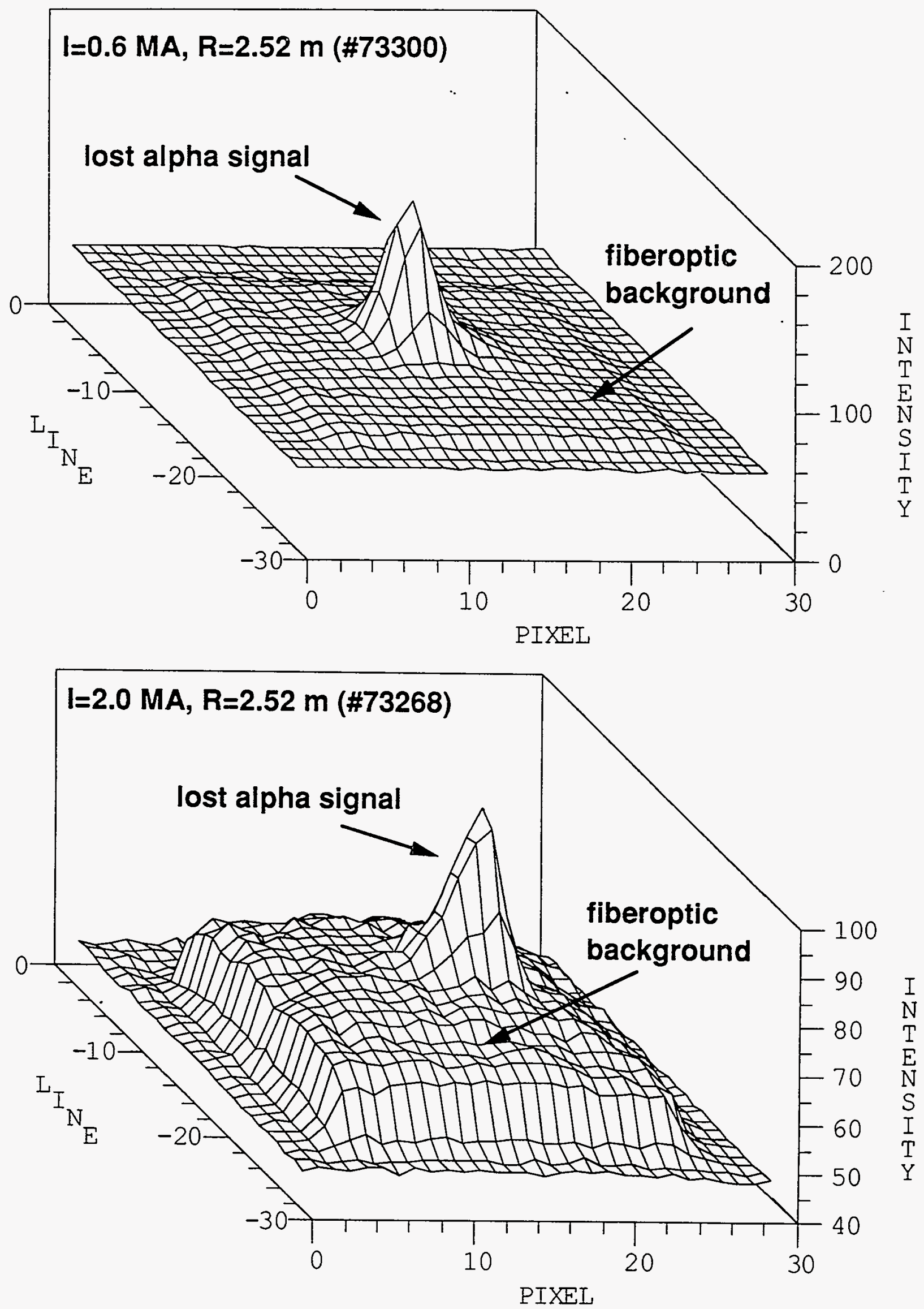

Fig. 3 


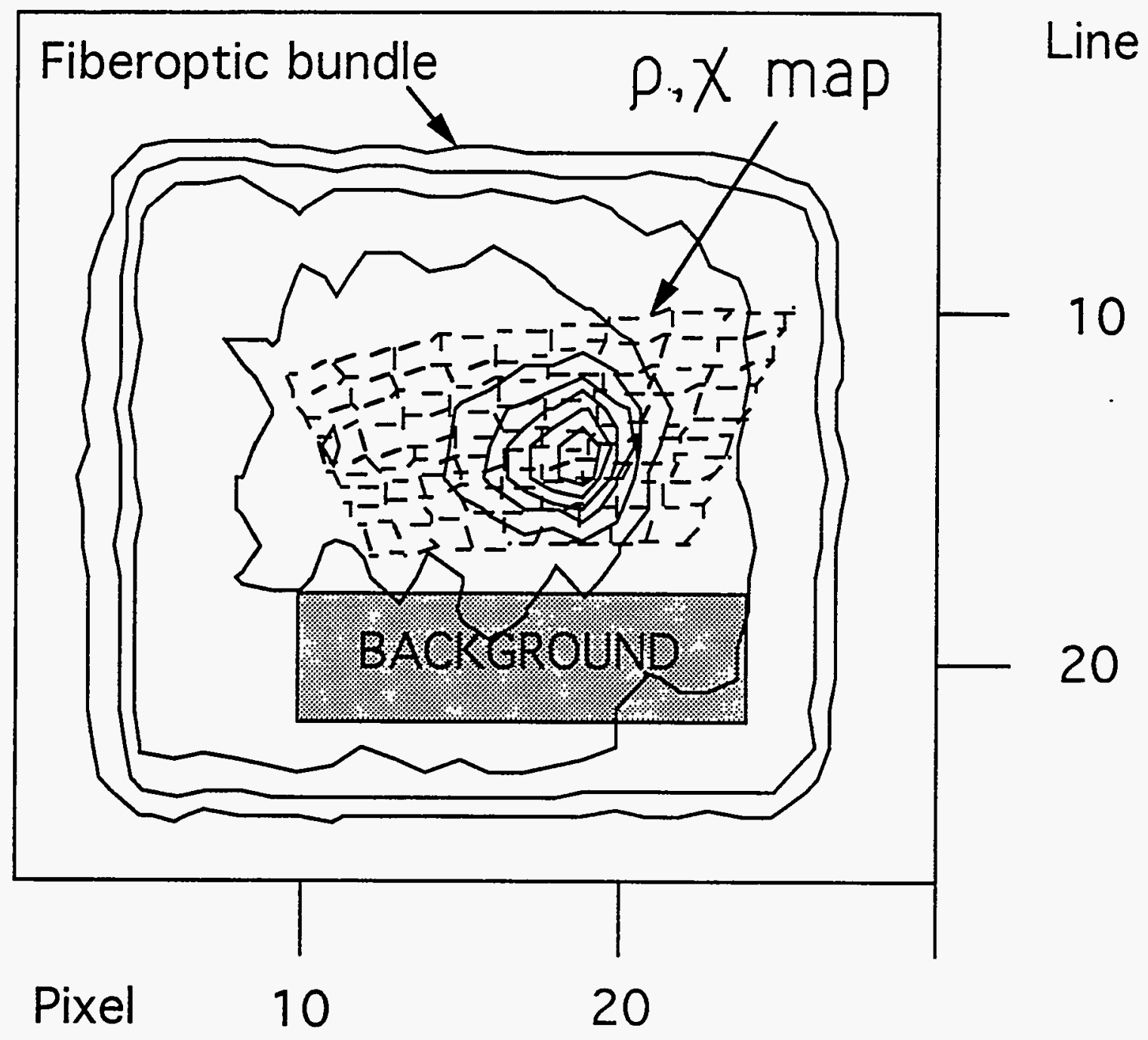

Fig. 4 

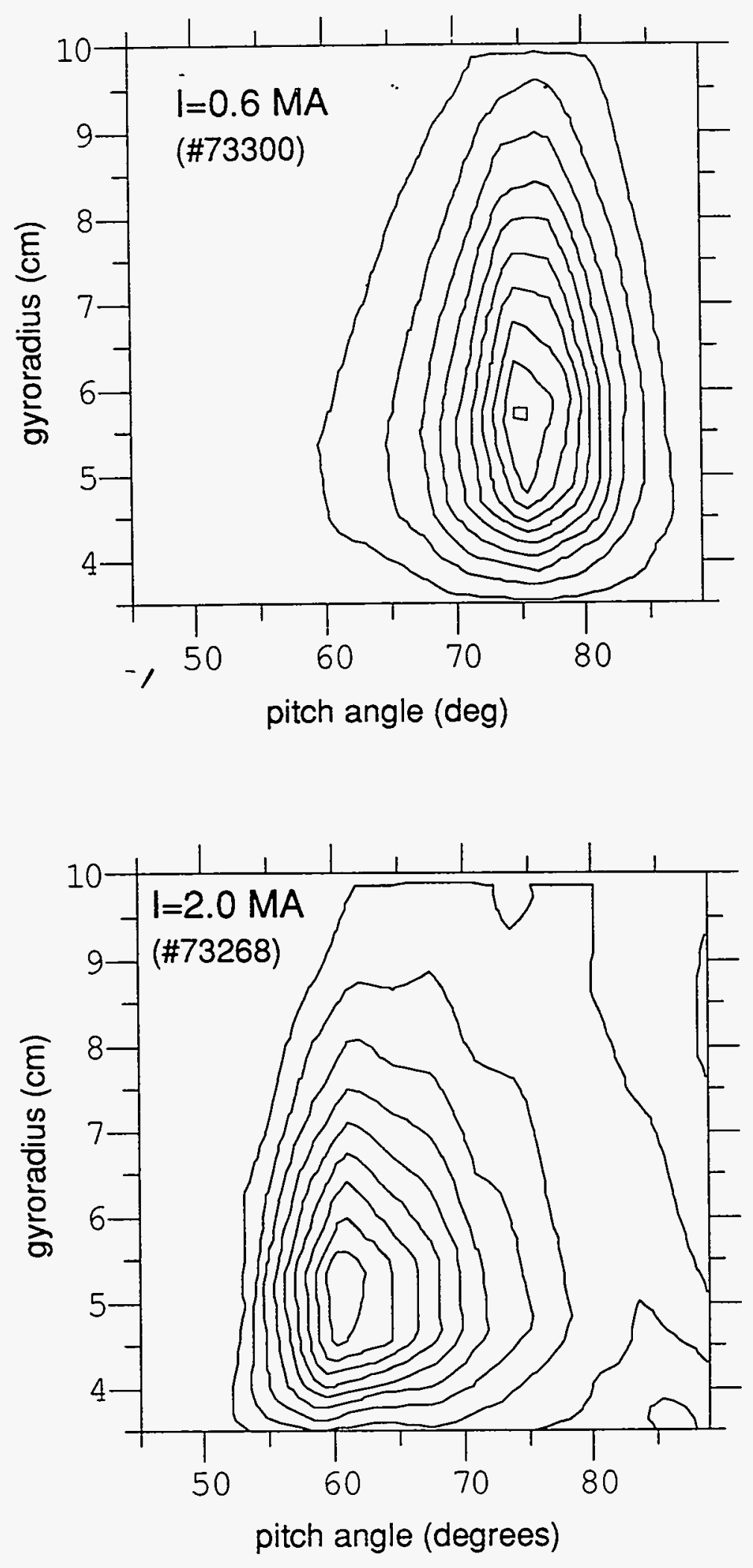

Fig. 5 


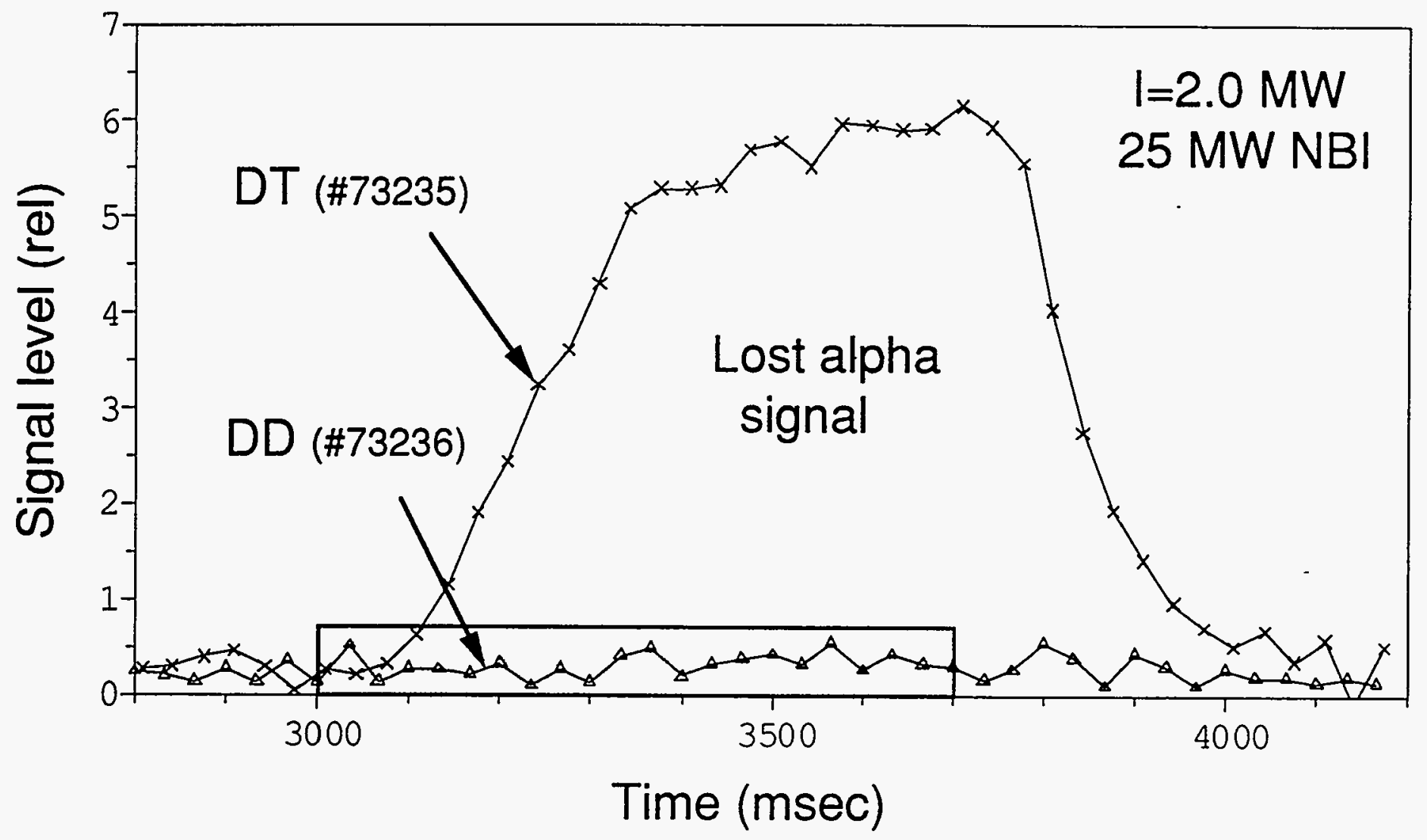

Fig. 6 

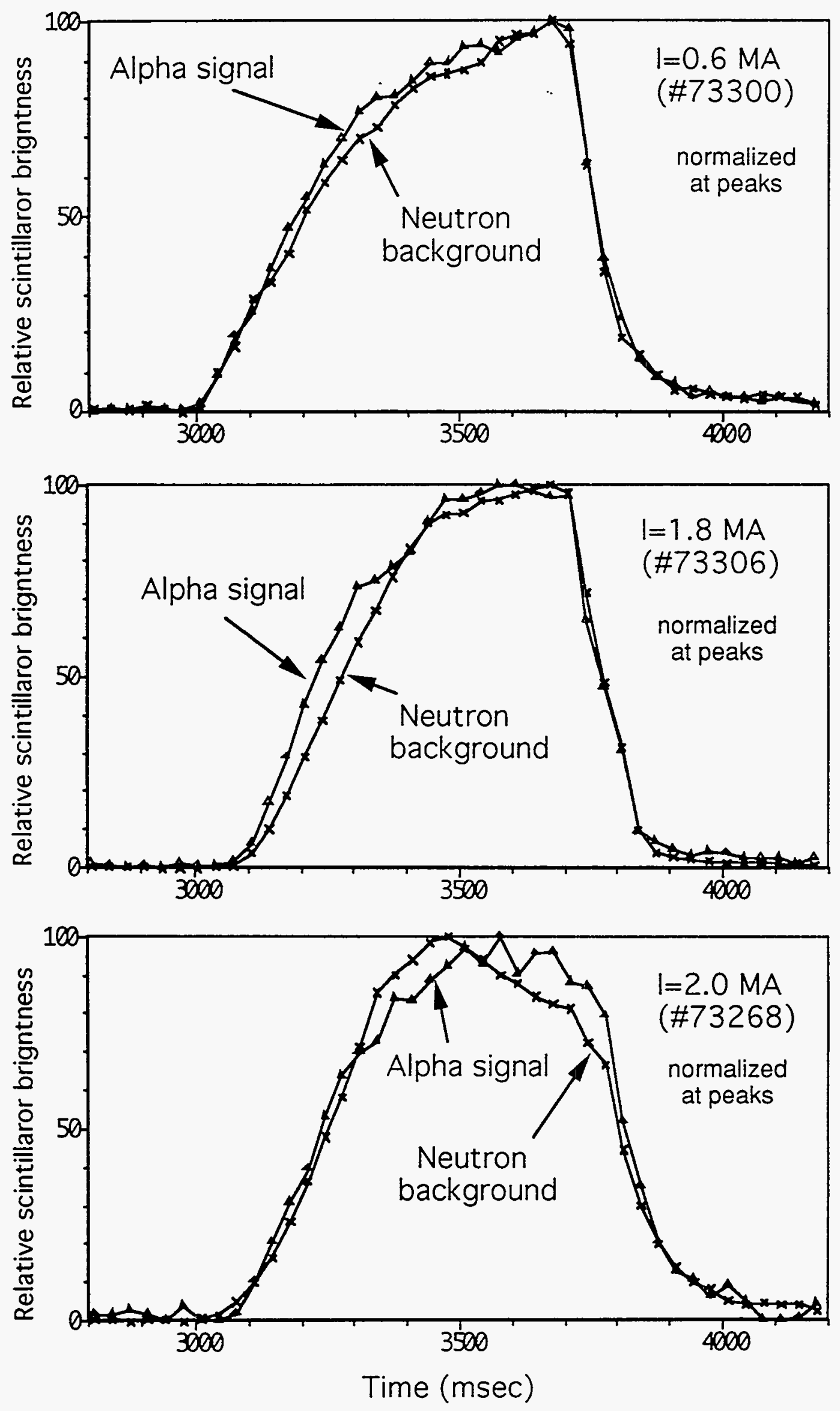

Fig. 7 

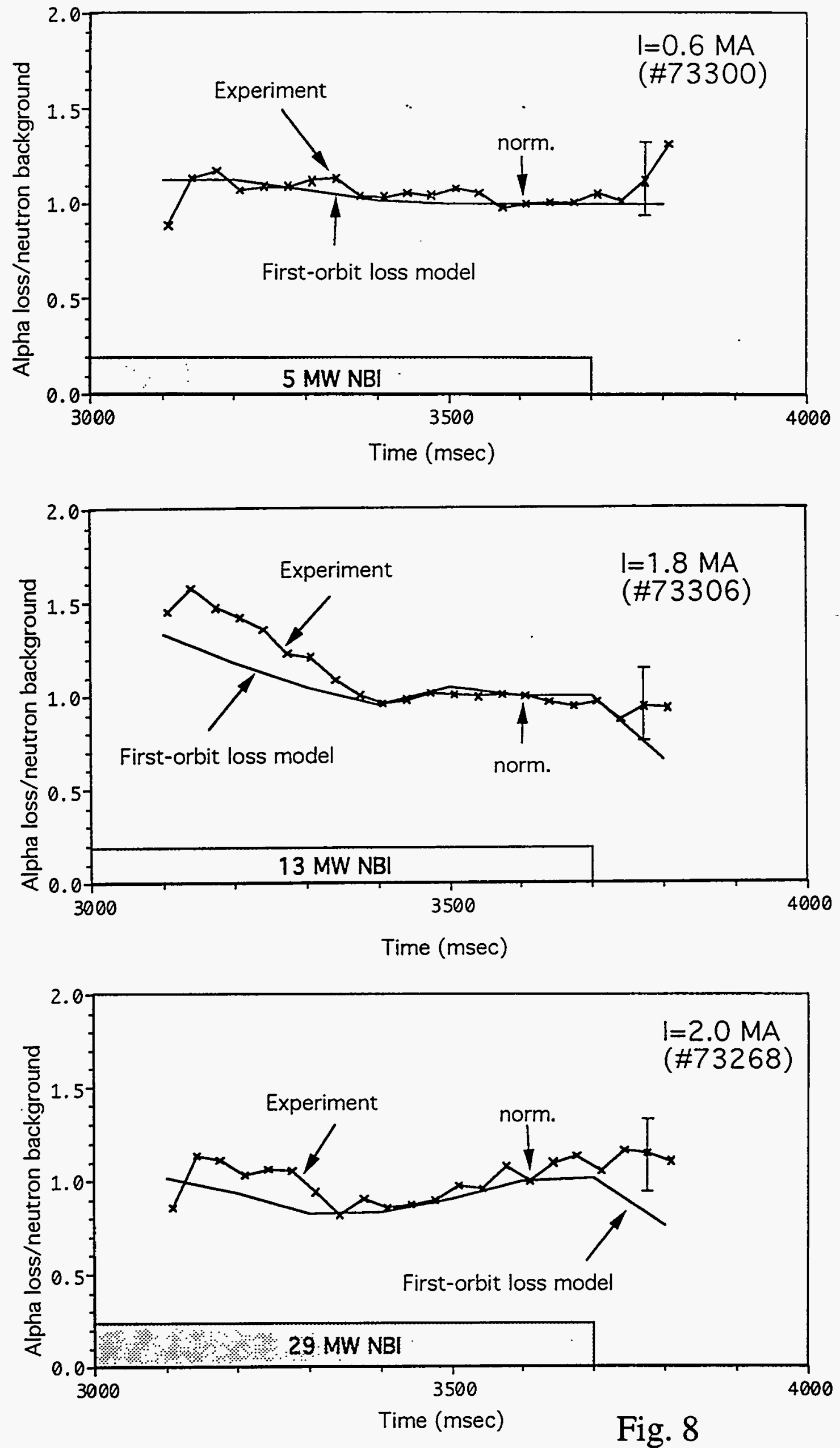


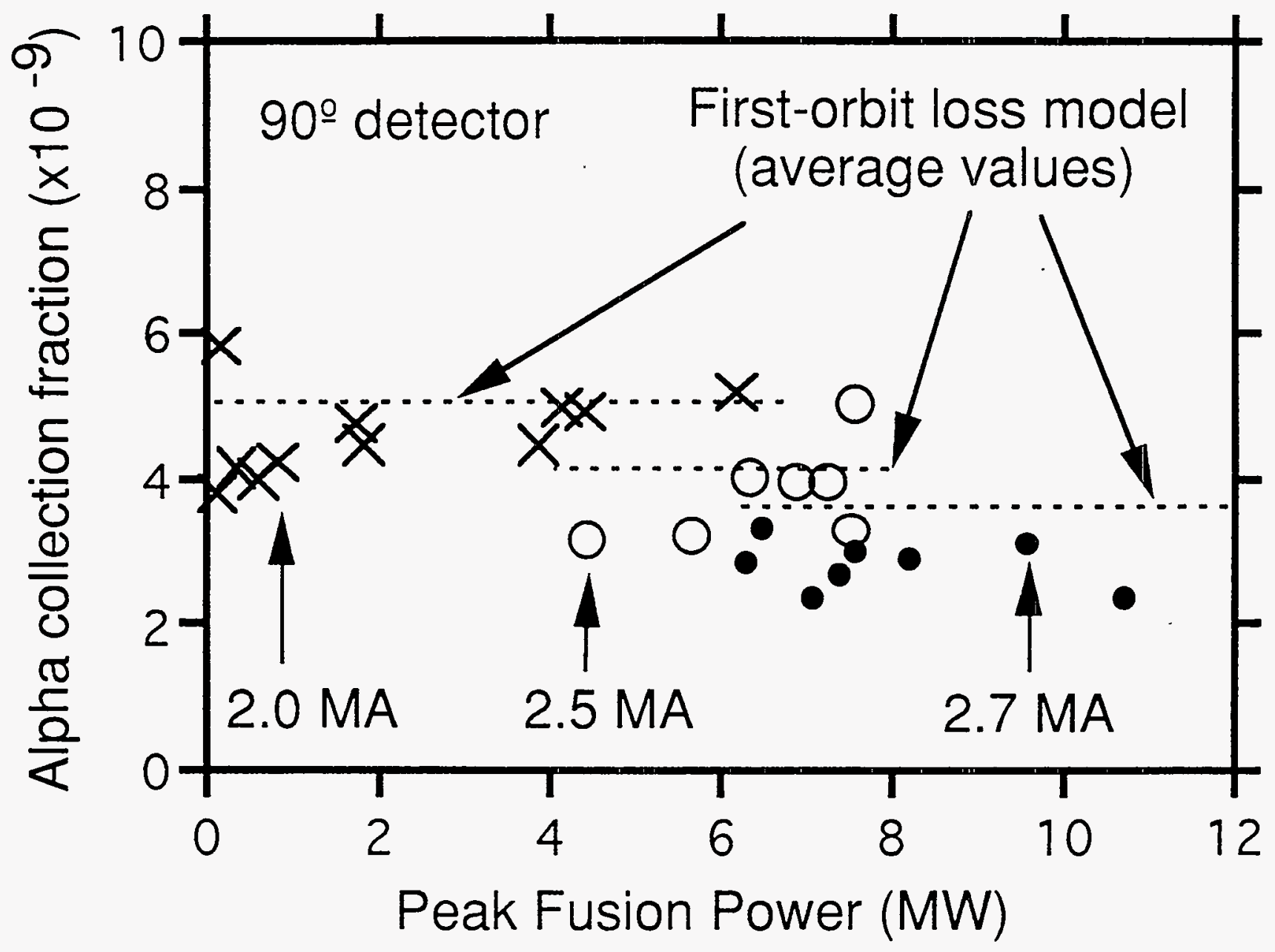

Fig. 9 


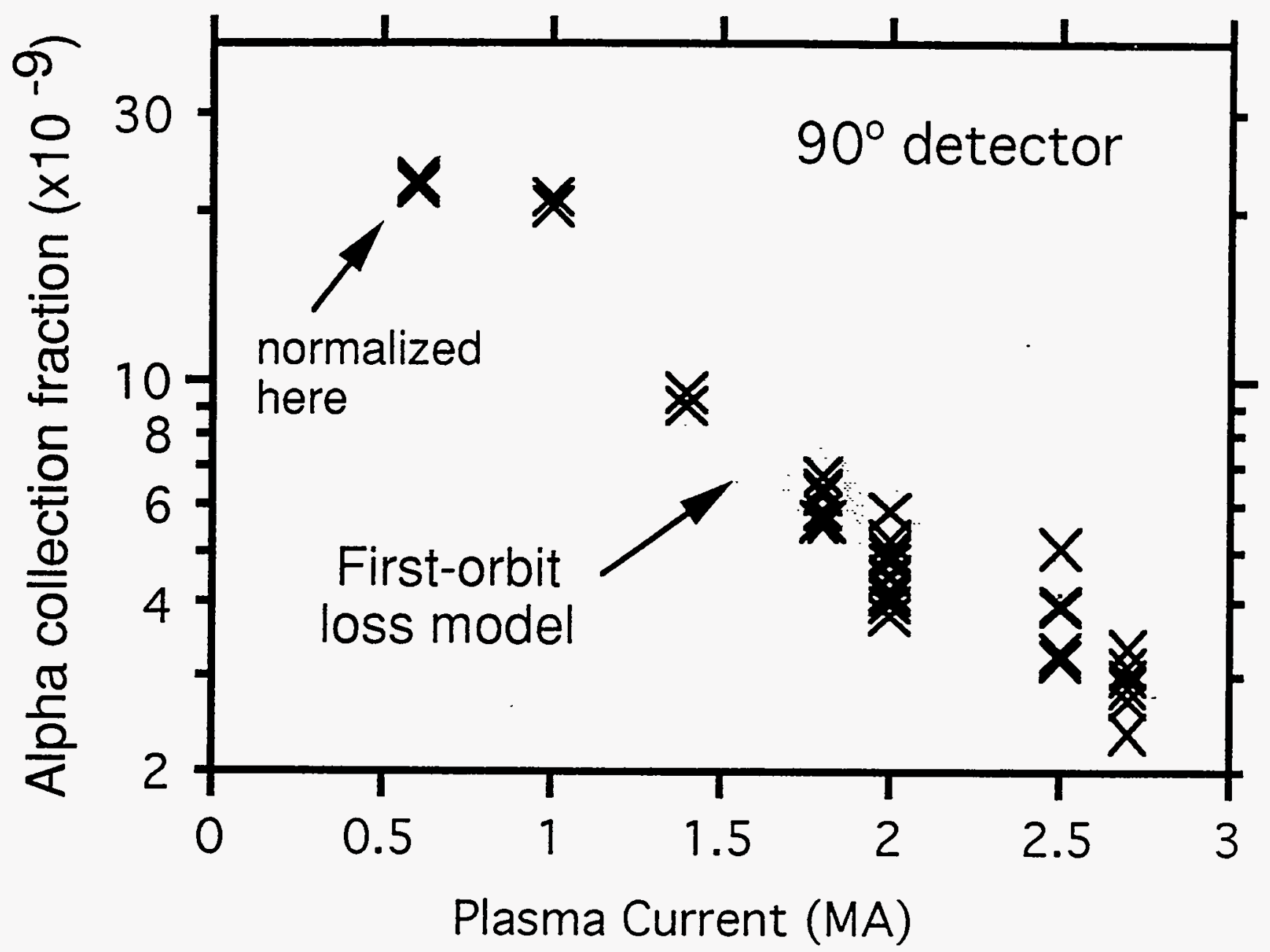

Fig. 10 

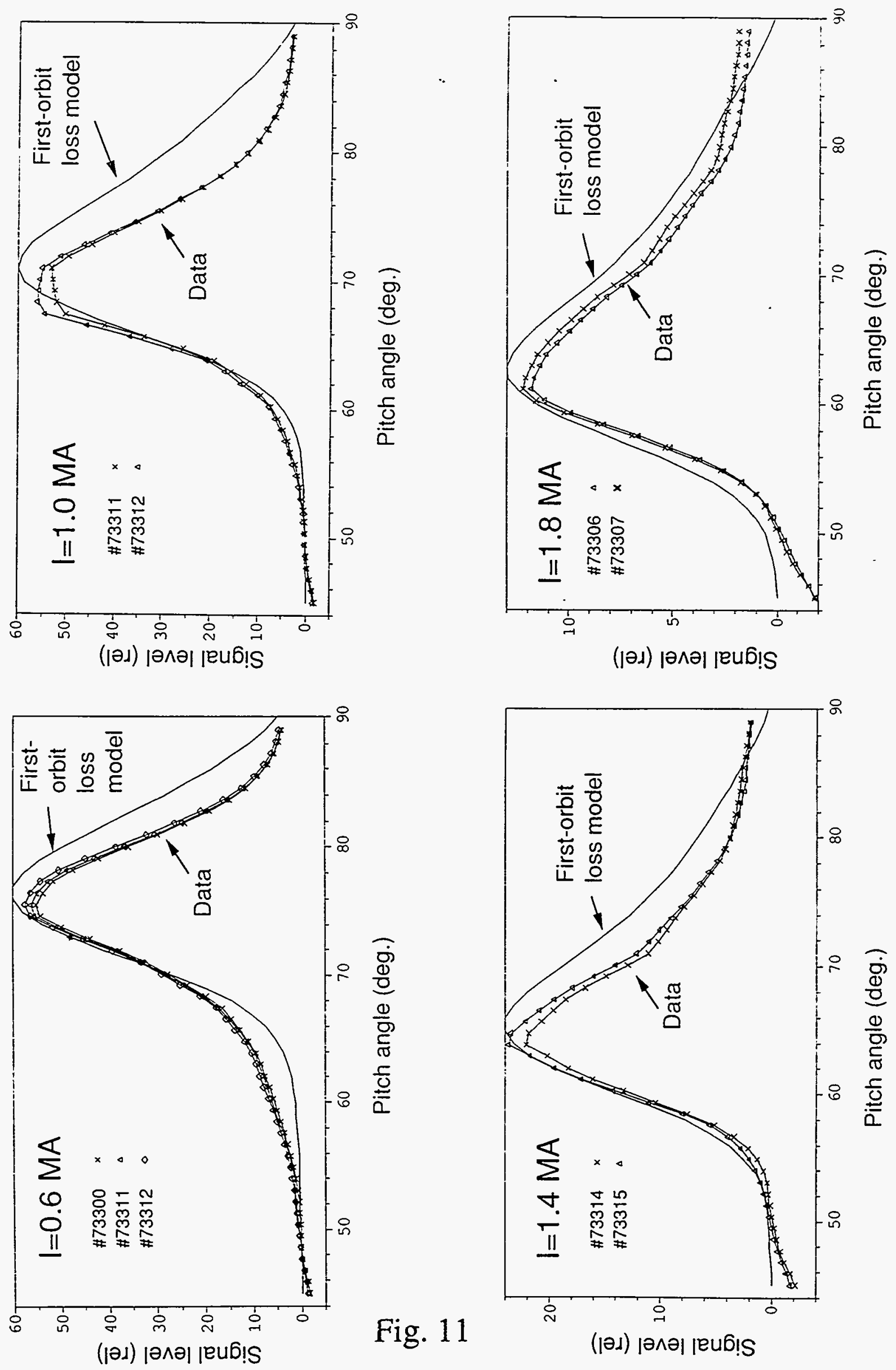

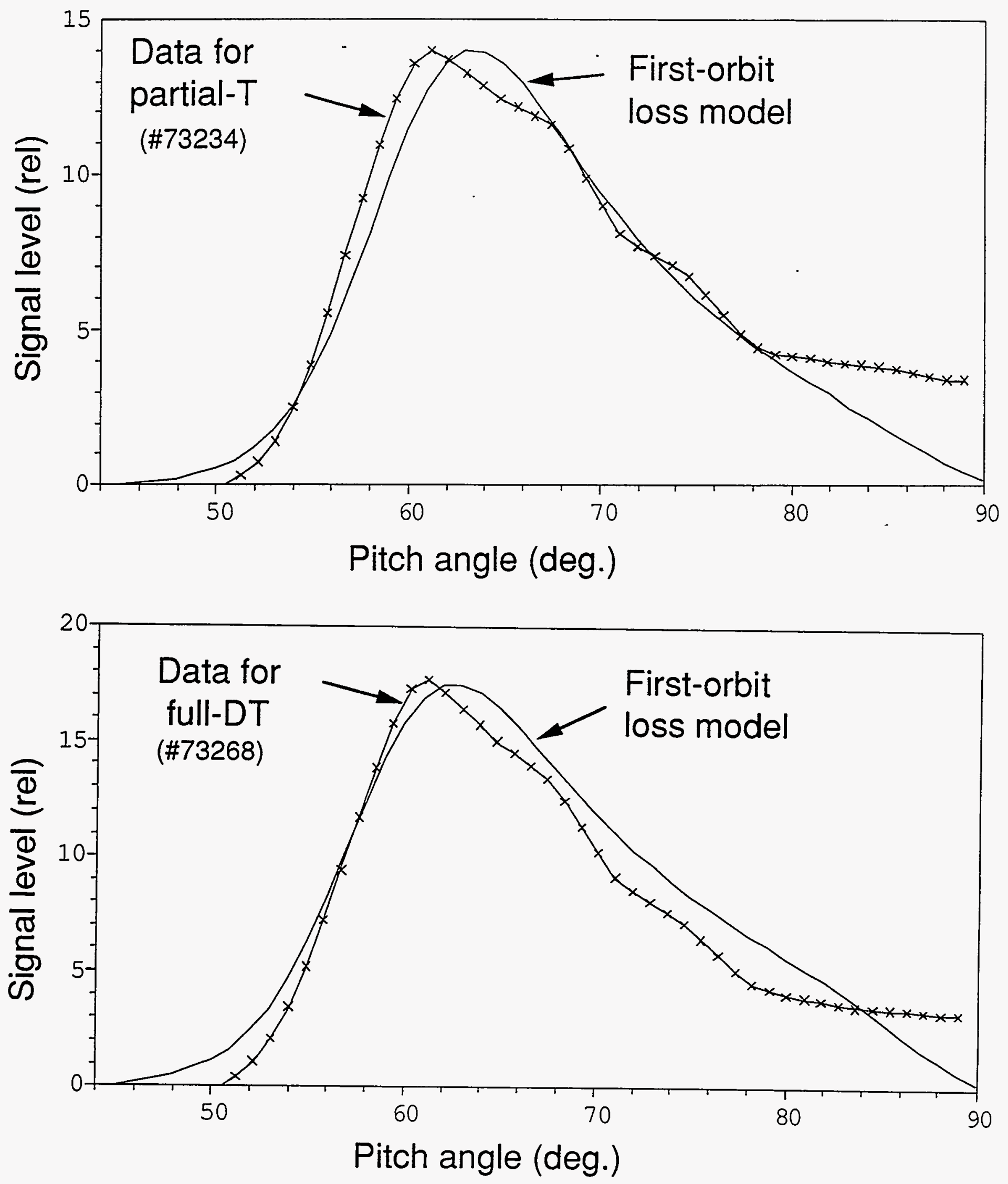

Fig. 12 


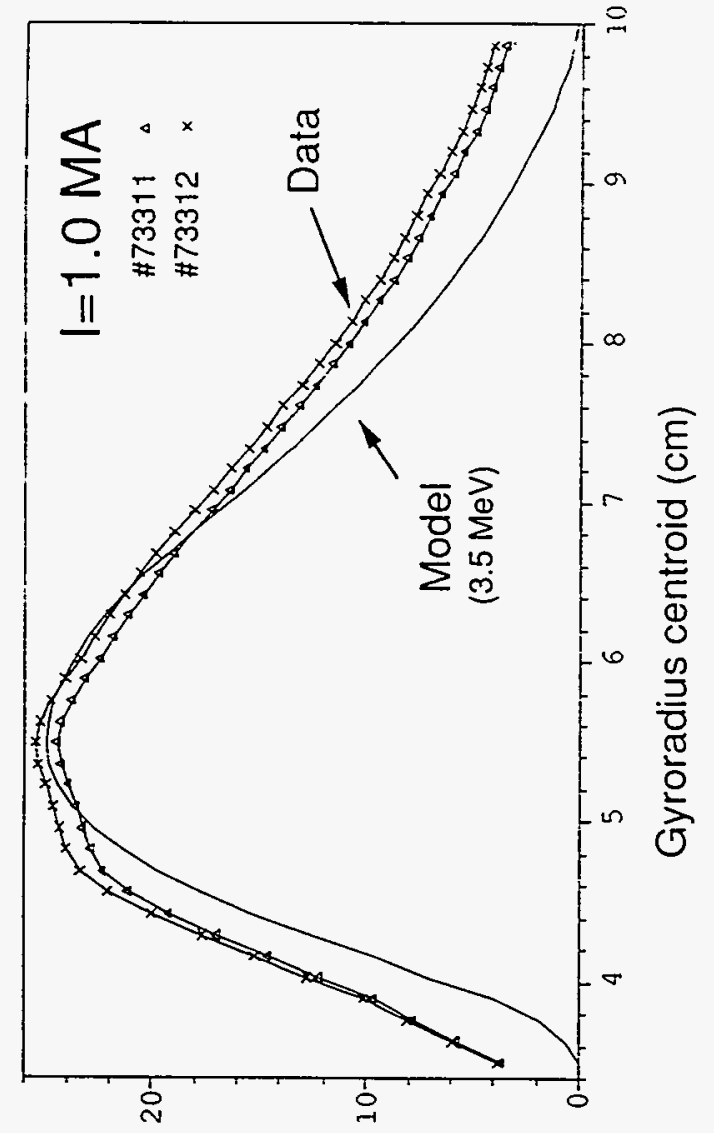

(ןد) |ә^ә| |еu6!S

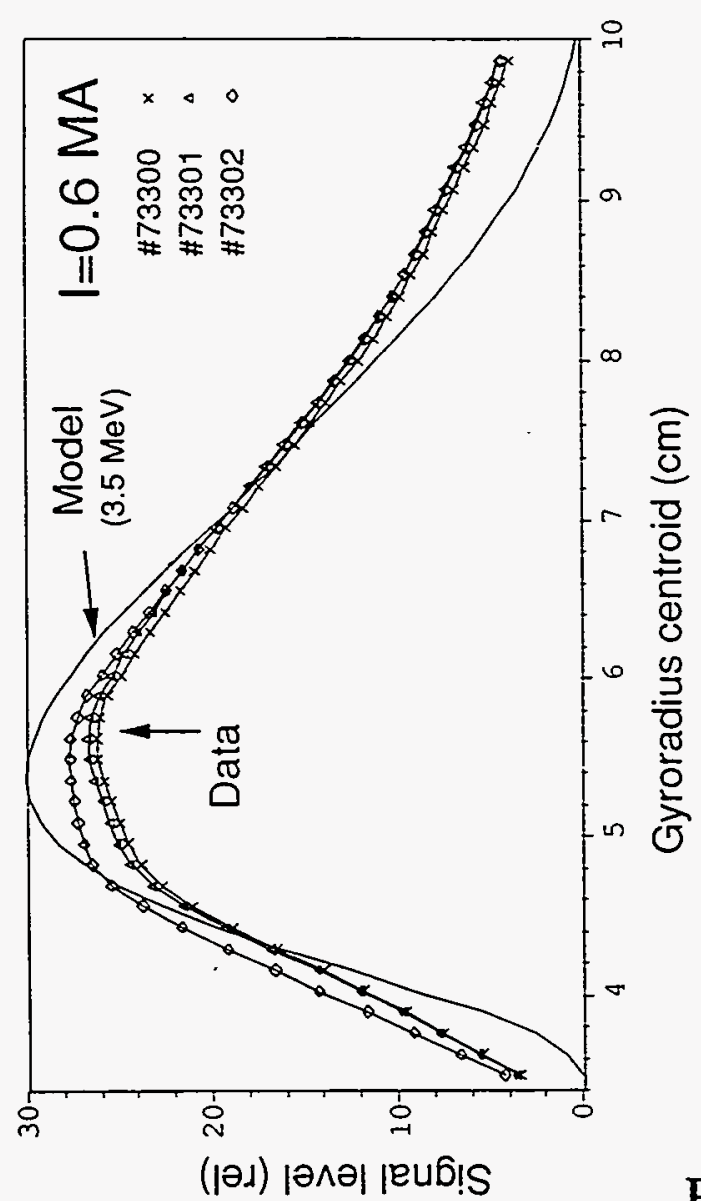

Fig. 13
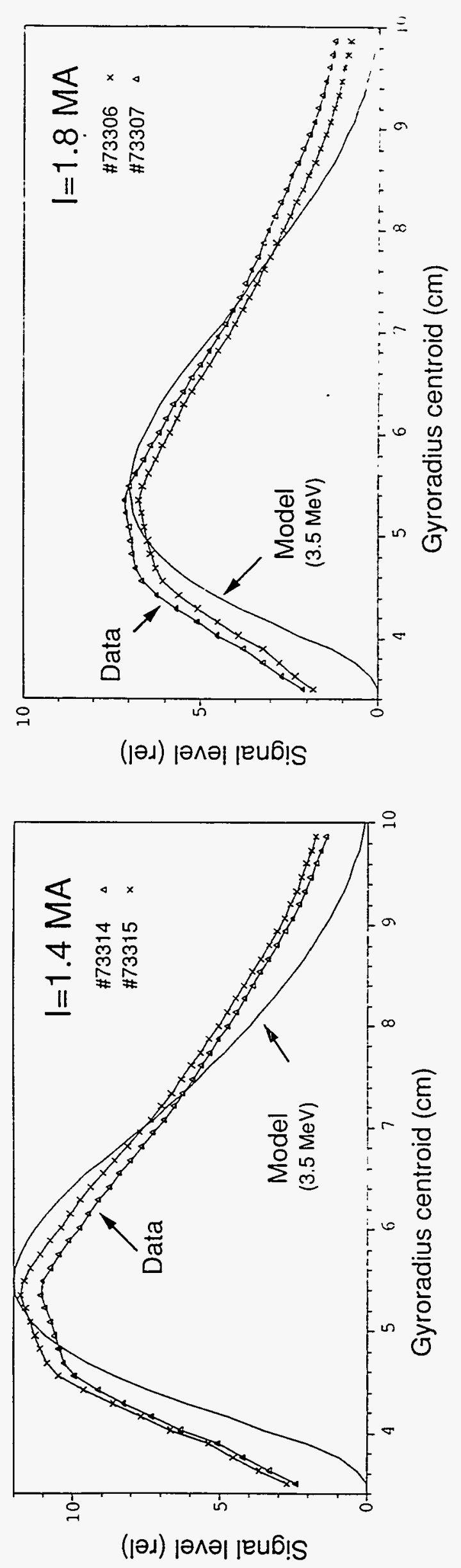


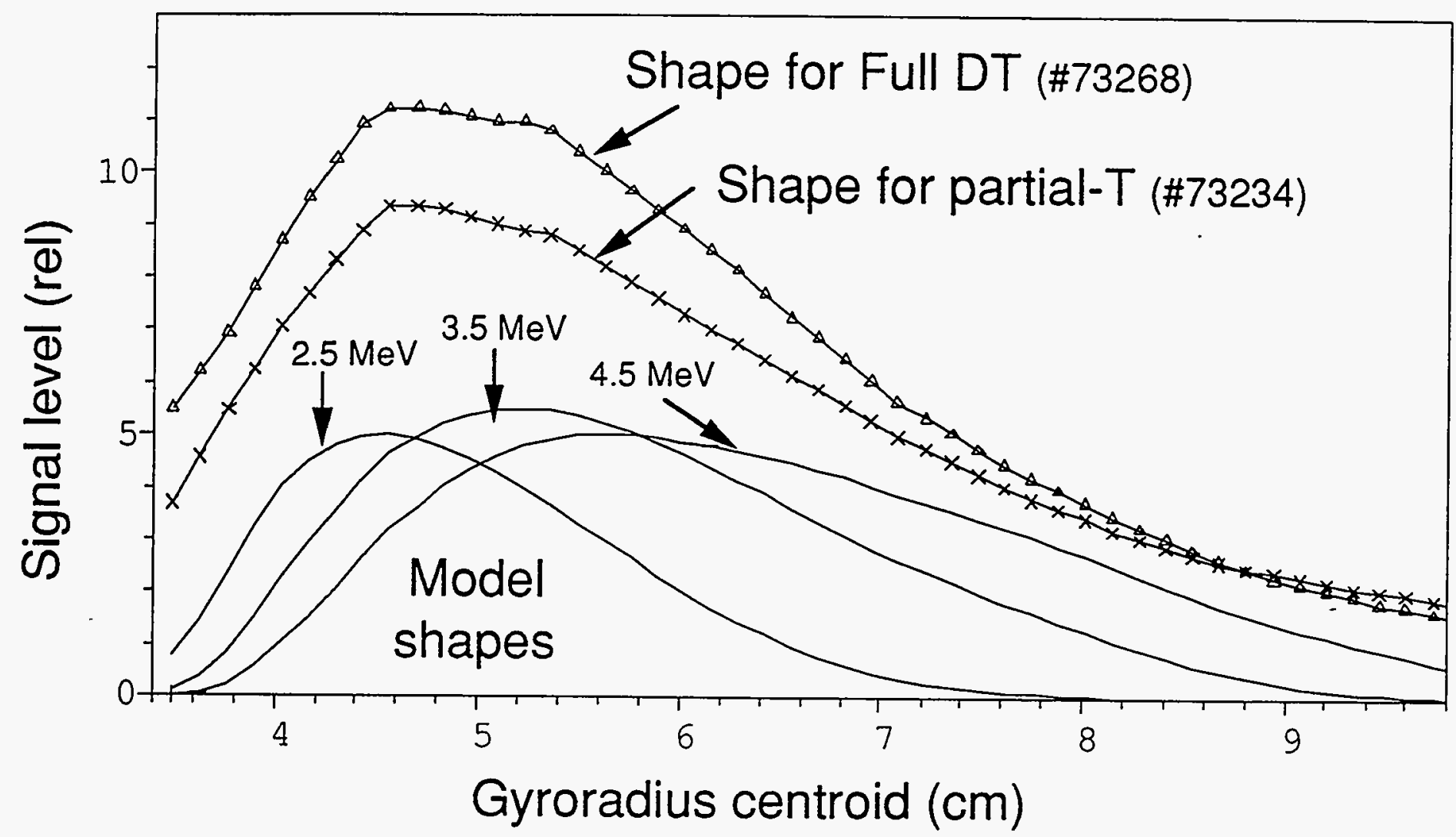

Fig. 14 

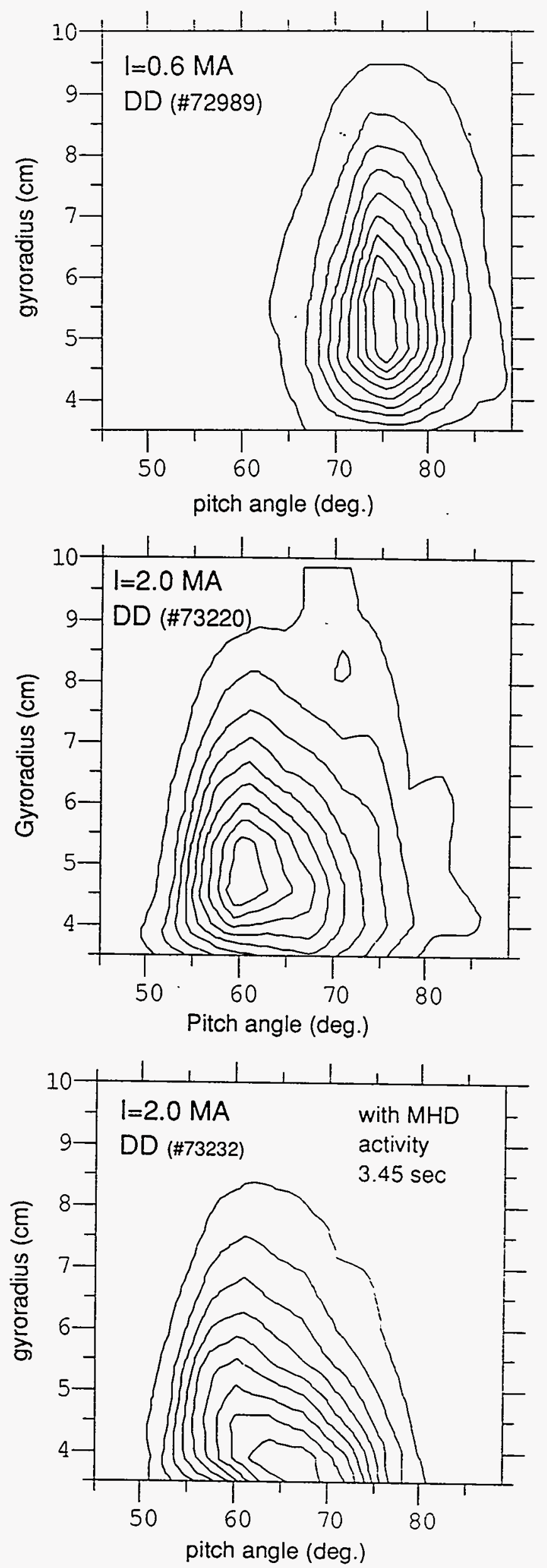

Fig. 15 

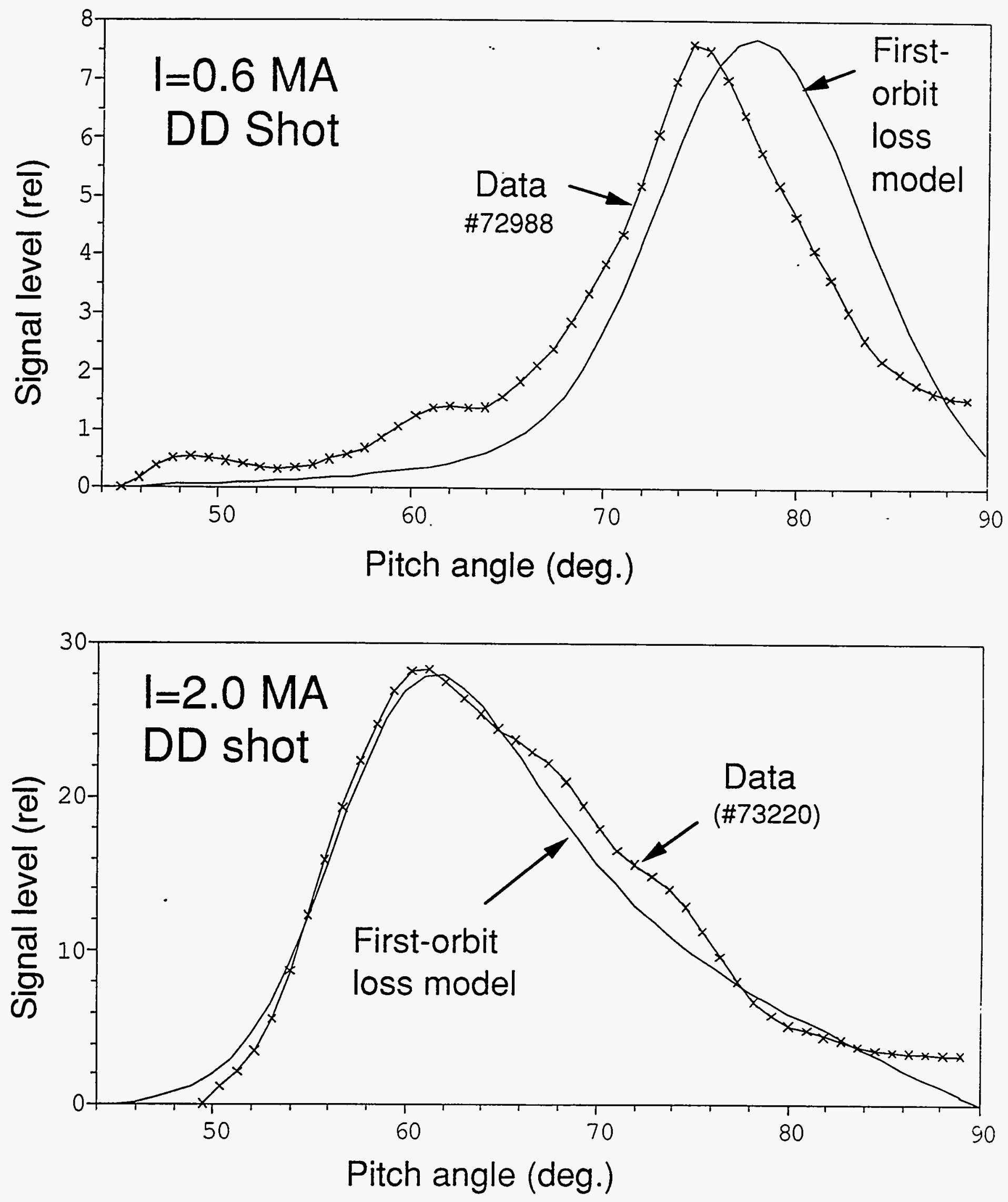

Fig. 16 

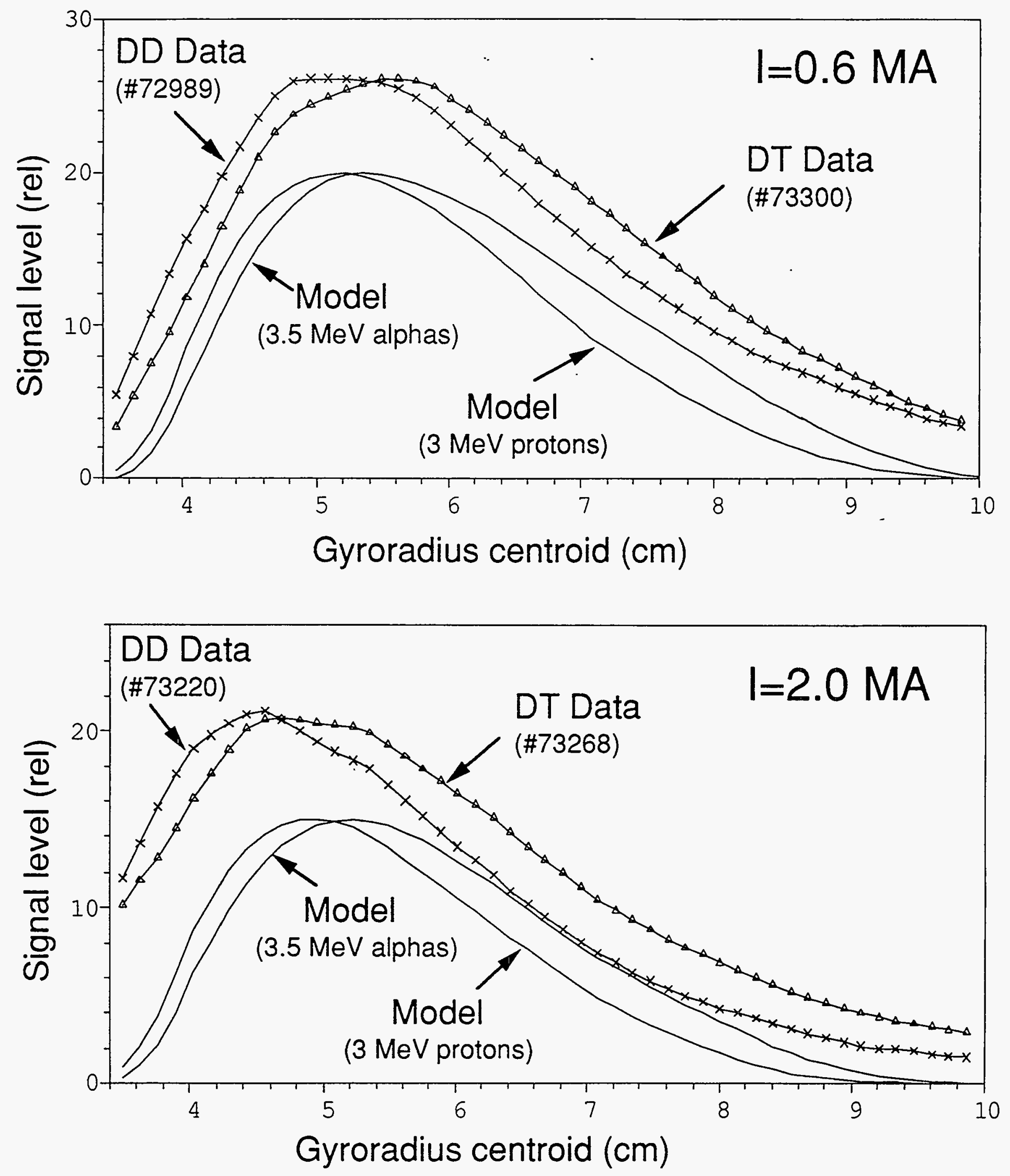

Fig. 17 


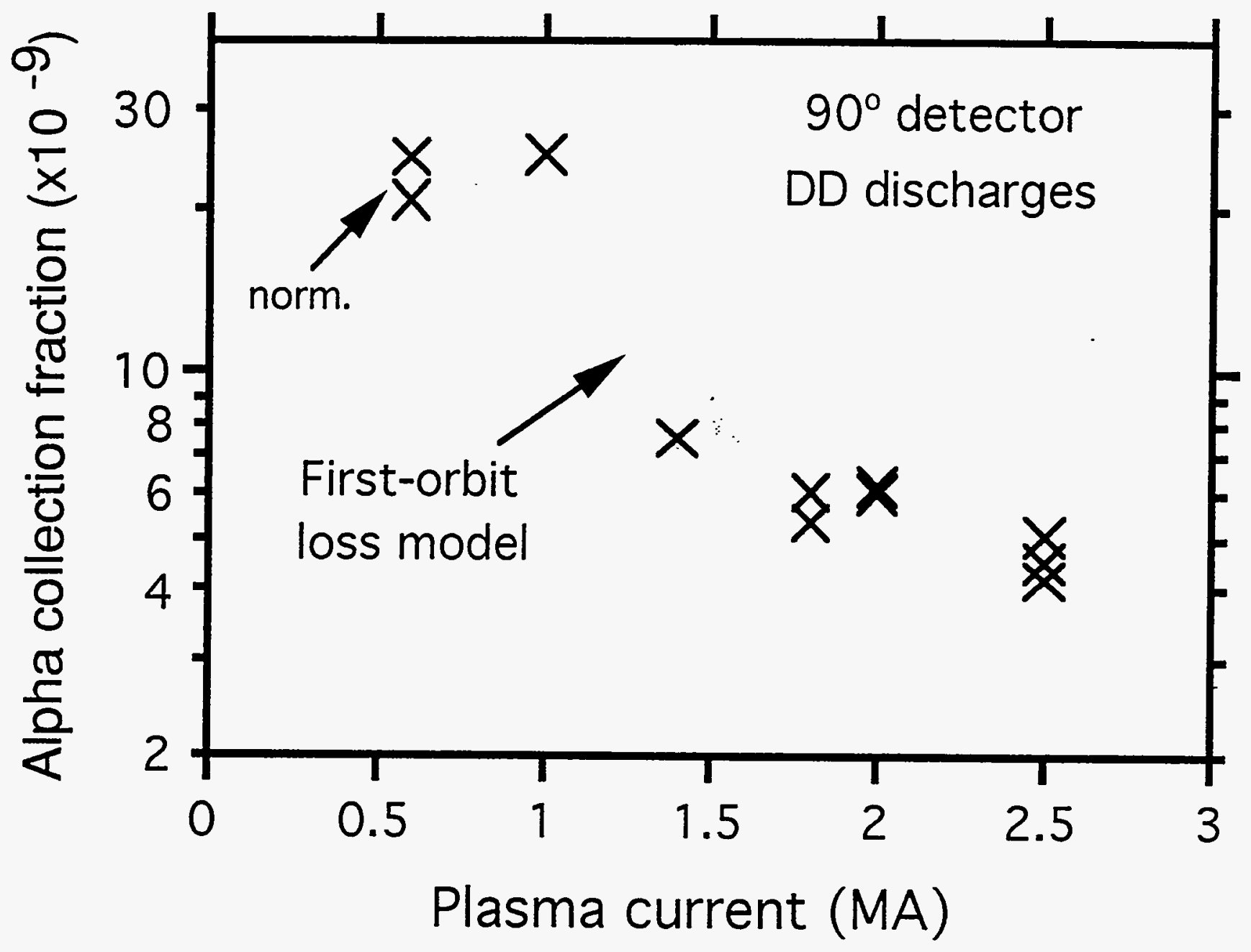

Fig. 18 

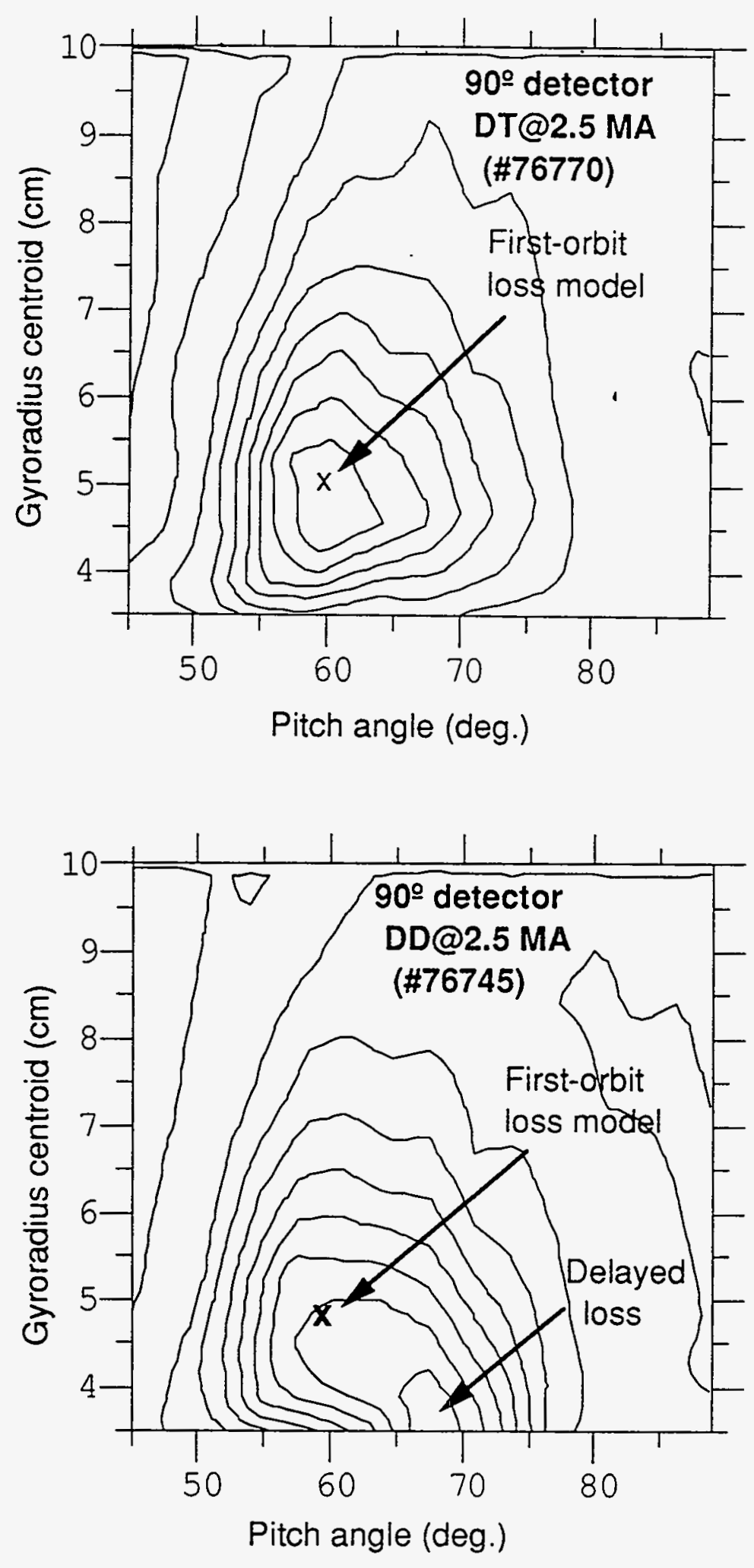

Fig. 19 

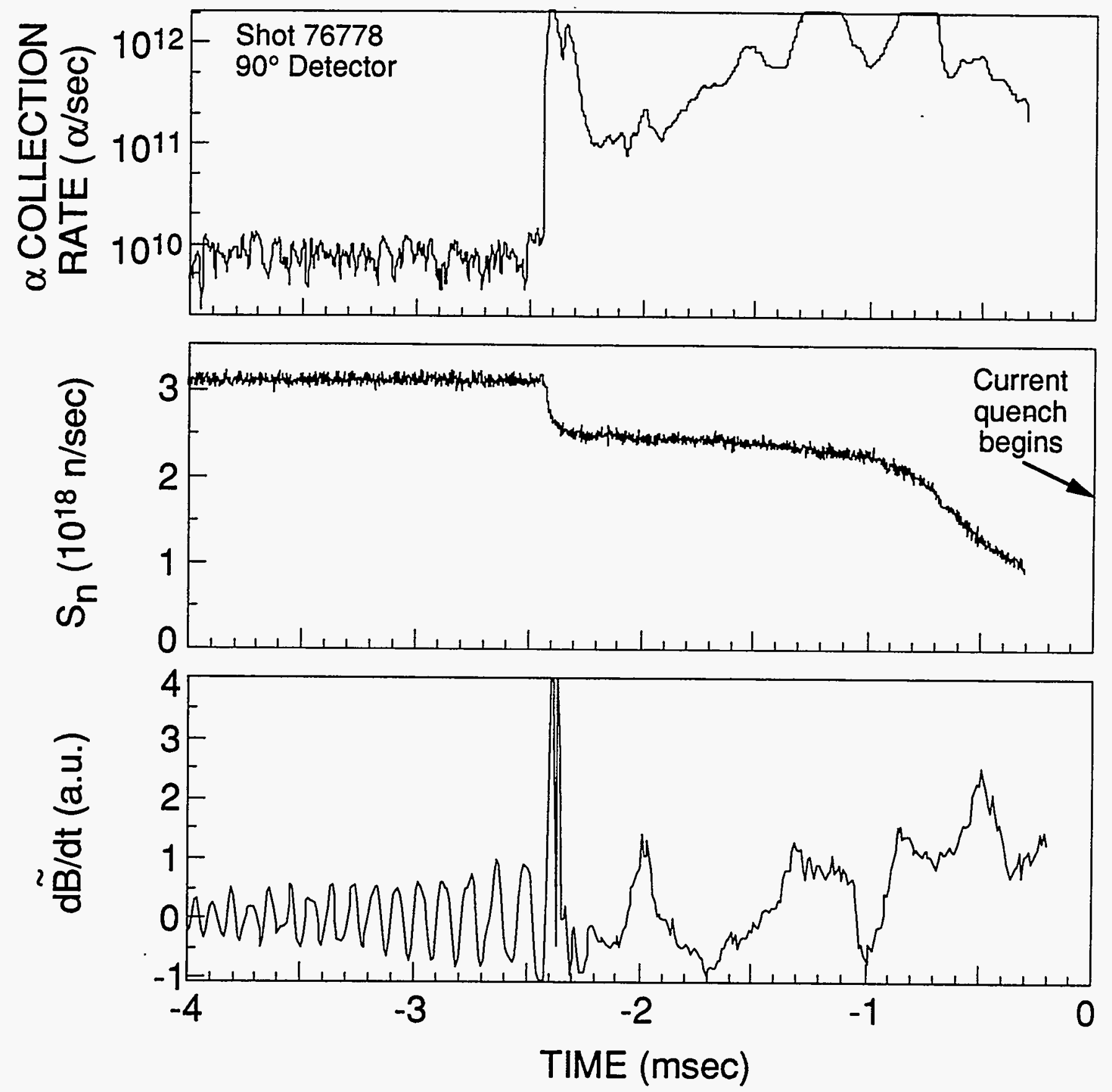

Fig. 20 

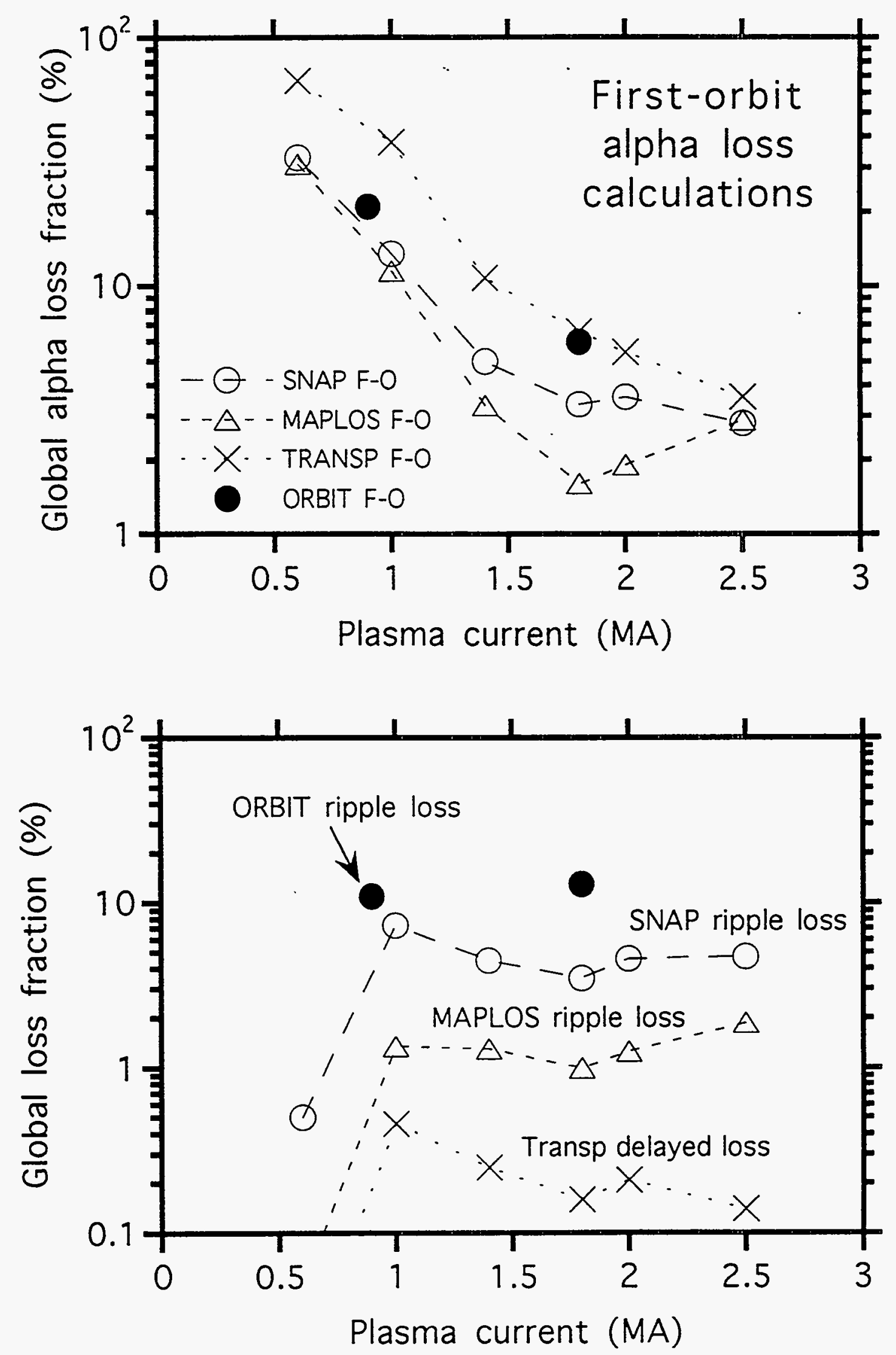

Fig. 21 
relative alpha loss

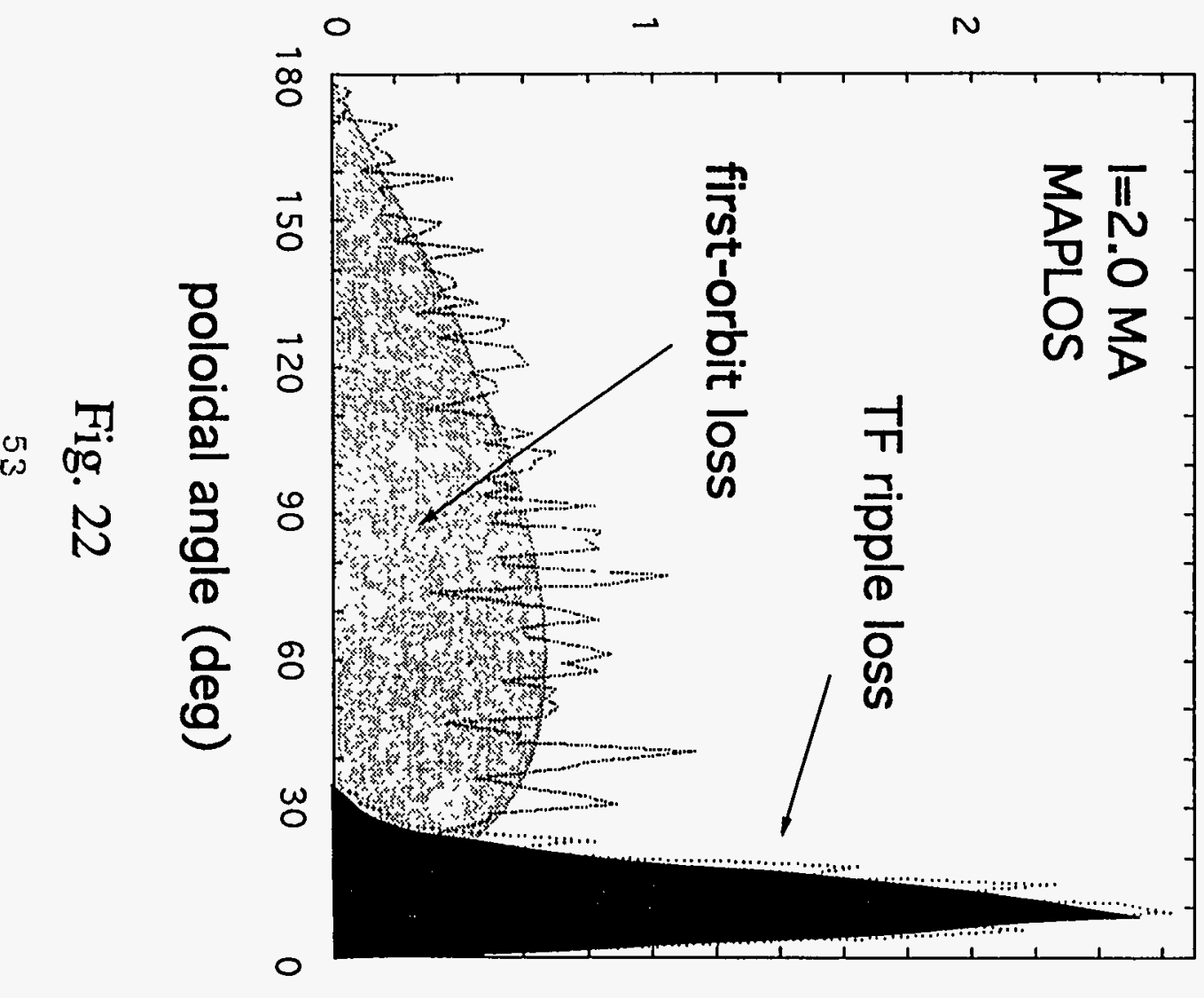

relative alpha loss

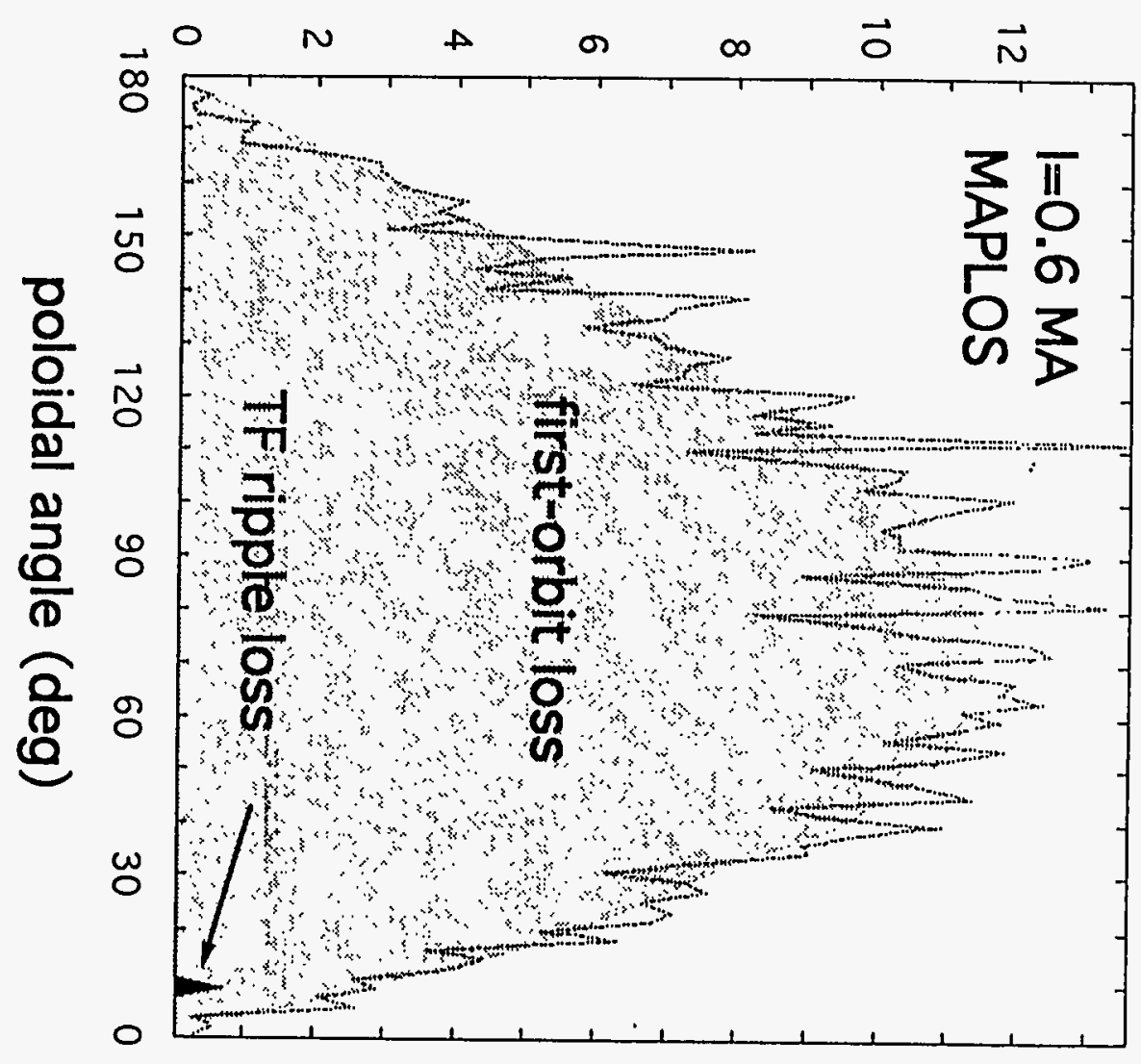




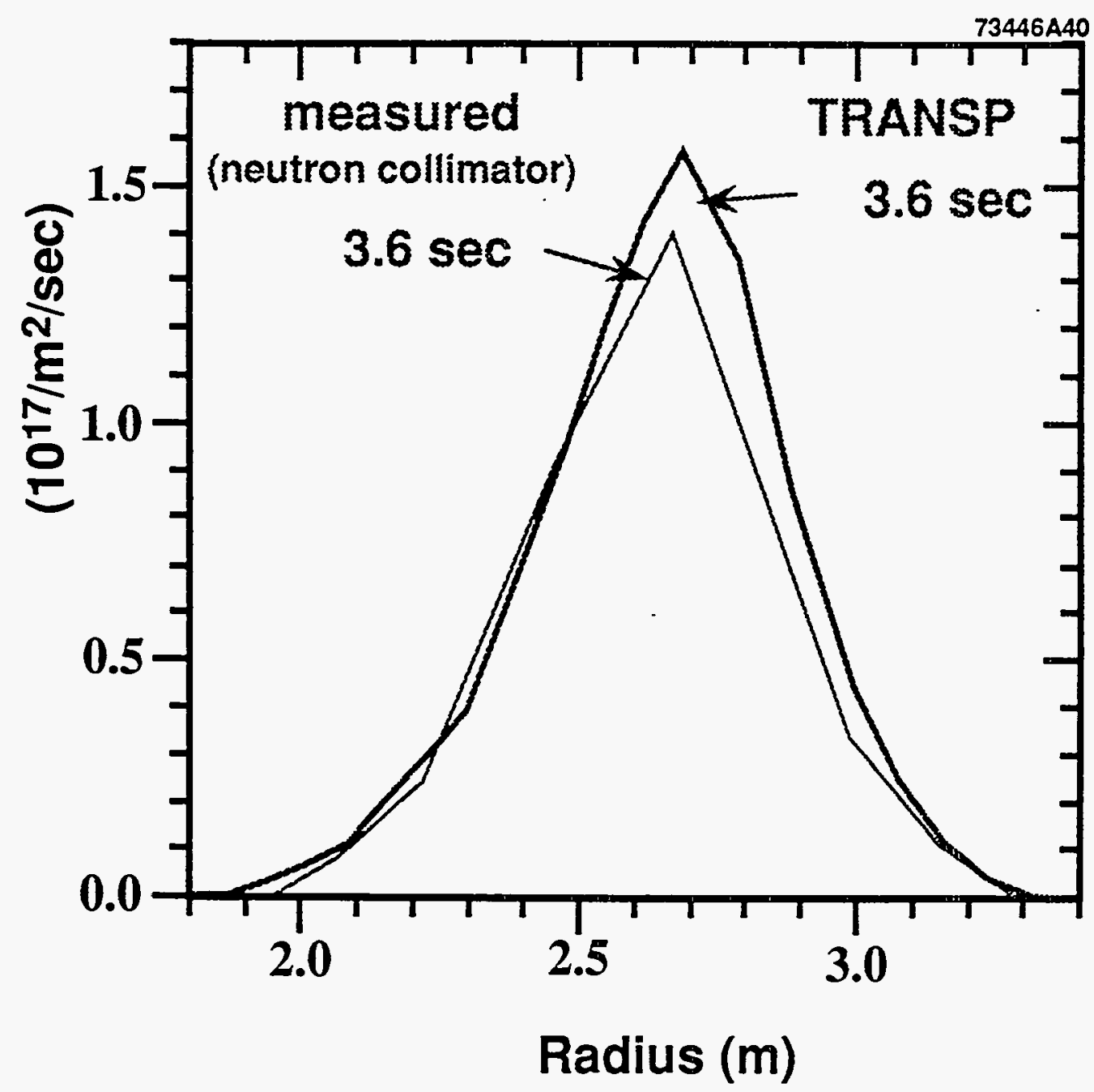

Fig. 23 

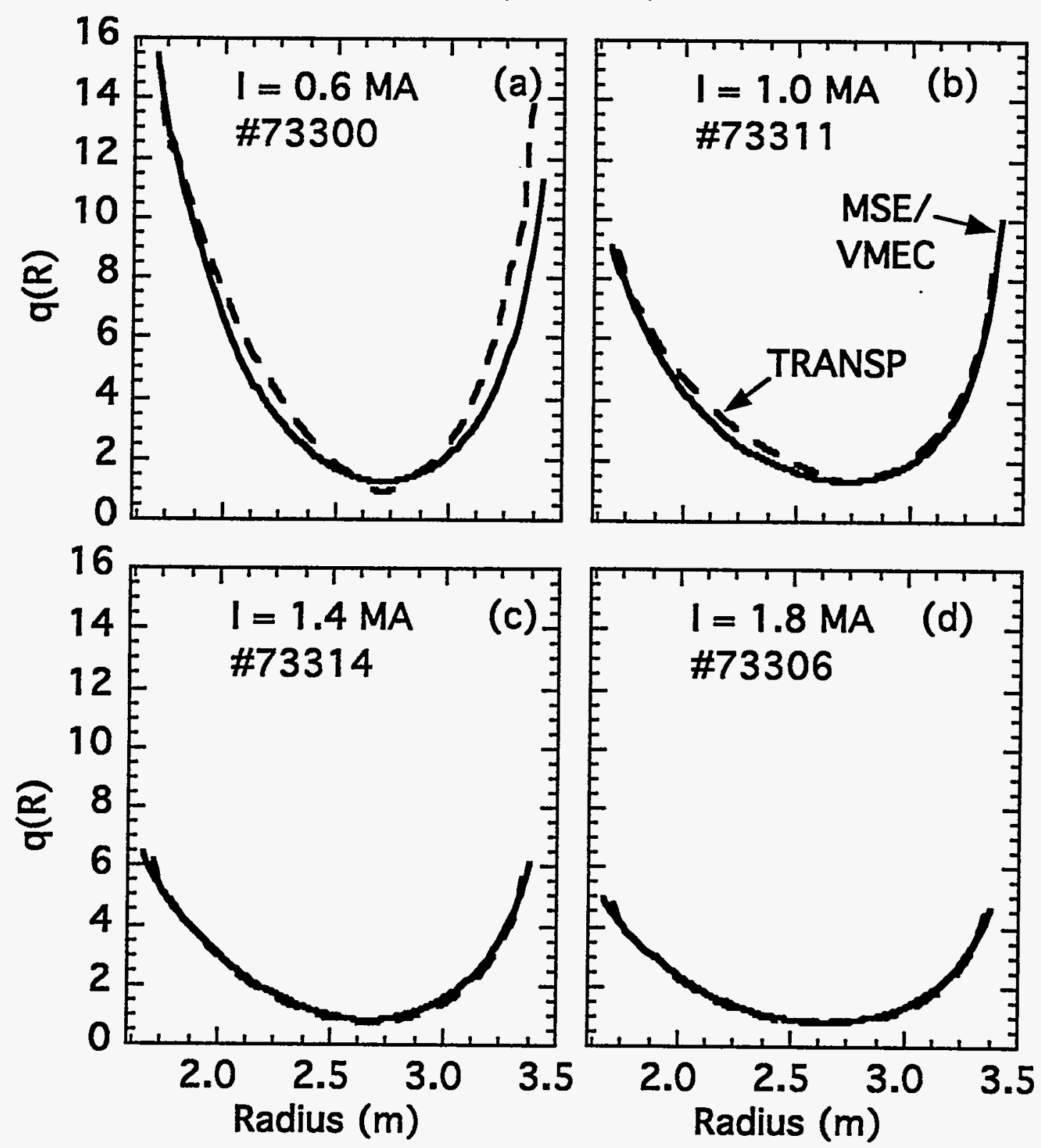

Fig. 24 


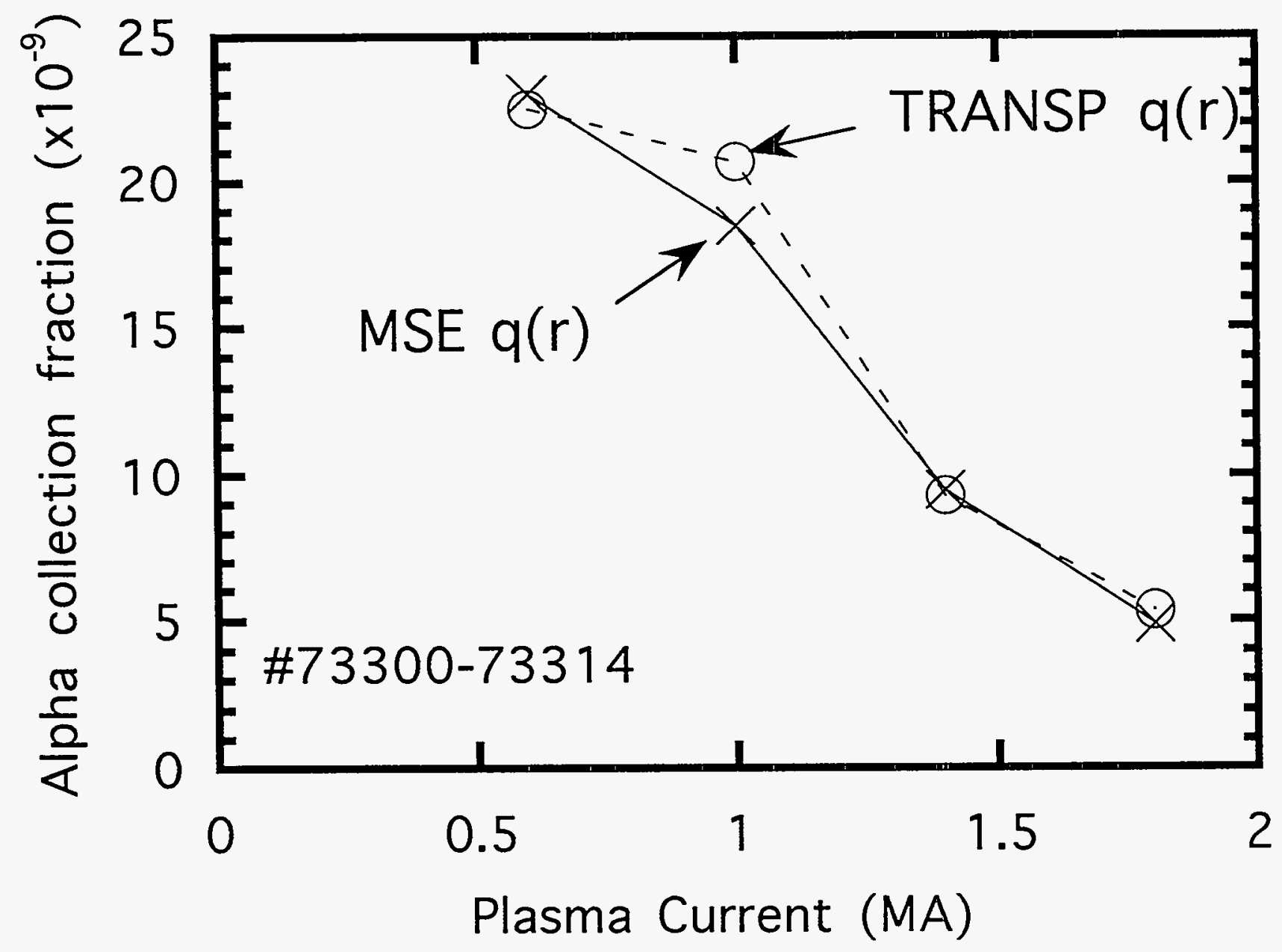

Fig. 25 


\section{EXTERNAL DISTRIBUTION IN ADDITION TO UC-420}

Dr. F. Paoloni, Univ. of Wollongong, AUSTRALIA

Prof. R.C. Cross, Univ. of Sydney, AUSTRALIA

Plasma Research Lab., Australian Nat. Univ., AUSTRALIA

Prof. I.R. Jones, Flinders Univ, AUSTRALIA

Prof. F. Cap, Inst. for Theoretical Physics, AUSTRIA

Prof. M. Heindler, Institut für Theoretische Physik, AUSTRIA

Prof. M. Goossens, Astronomisch Instituut, BELGIUM

Ecole Royale Militaire, Lab. de Phy. Plasmas, BELGIUM

Commission-European, DG. XII-Fusion Prog., BELGIUM

Prof. R. Bouciqué, Rijksuniversiteit Gent, BELGIUM

Dr. P.H. Sakanaka, Instituto Fisica, BRAZIL

Prof. Dr. I.C. Nascimento, Instituto Fisica, Sao Paulo, BRAZIL Instituto Nacional De Pesquisas Espaciais-INPE, BRAZIL

Documents Office, Atomic Energy of Canada Ltd., CANADA

Ms. M. Morin, CCFMTokamak de Varennes, CANADA

Dr. M.P. Bachynski, MPB Technologies, Inc., CANADA

Dr. H.M. Skarsgard, Univ. of Saskatchewan, CANADA

Prof. J. Teichmann, Univ. of Montrea!, CANADA

Prof. S.R. Sreenivasan, Univ. of Calgary, CANADA

Prof. T.W. Johnston, INRS-Energie, CANADA

Dr. R. Bolton, Centre canadien de fusion magnétique, CANADA

Dr. C.R. James, Univ. of Alberta, CANADA

Dr. P. Lukác, Komenského Universzita, CZECHO-SLOVAKIA

The Librarian, Culham Laboratory, ENGLAND

Library, R61, Rutherford Appleton Laboratory. ENGLAND

Mrs. S.A. Hutchinson, JET Library, ENGLAND

Dr. S.C. Sharma, Univ. of South Pacific, FIJI ISLANDS

P. Mähönen, Univ, of Helsinki, FINLAND

Prof. M.N. Bussac, Ecole Polytechnique, FRANCE

C. Mouttet, Lab. de Physique des Milieux lonisés, FRANCE

J. Radet, CEN/CADARACHE - Bat 506, FRANCE

Prof. E. Economou, Univ. of Crete, GREECE

Ms. C. Rinni, Univ. of loannina, GREECE

Preprint Library, Hungarian Academy of Sci., HUNGARY

Dr. B. DasGupta, Saha inst. of Nuclear Physics, INDIA

Dr. P. Kaw, Inst. for Plasma Research, INDIA

Dr. P. Rosenau, Israel Inst. of Technology, ISRAEL

Librarian, Intemational Center for Theo Physics, ITALY

Miss C. De Palo, Associazione EURATOM-ENEA, ITALY

Dr. G. Grosso, Istituto di Fisica del Plasma, ITALY

Prof. G. Rostangni, Istituto Gas Ionizzati Del Cnr, ITALY
Dr. H. Yamato, Toshiba Res \& Devel Center, JAPAN

Prof. I. Kawakami, Hiroshima Univ., JAPAN

Prof. K. Nishikawa, Hiroshima Univ., JAPAN

Librarian, Naka Fusion Research Establishment, JAERI, JAPAN

Director, Japan Atomic Energy Research Inst., JAPAN

Prof. S. Itoh, Kyushu Univ., JAPAN

Research Info. Ctr., National Instit. for Fusion Science, JAPAN

Prof. S. Tanaka, Kyoto Univ., JAPAN

Library, Kyoto Univ., JAPAN

Prof. N. Inoue, Univ. of Tokyo, JAPAN

Secretary, Plasma Section, Electrotechnical Lab., JAPAN

Dr. O. Mitarai, Kumamoto Inst. of Technology, JAPAN

Dr. G.S. Lee, Korea Basic Sci. Crr., KOREA

J. Hyeon-Sook, Korea Atomic Energy Research Inst., KOREA

D.I. Choi, The Korea Adv. Inst. of Sci. \& Tech., KOREA

Prof. B.S. Liley, Univ. of Waikato, NEW ZEALAND

Inst of Physics, Chinese Acad Sci PEOPLE'S REP. OF CHINA

Library, Inst. of Plasma Physics, PEOPLE'S REP. OF CHINA

Tsinghua Univ. Library, PEOPLE'S REPUBLIC OF CHINA

Z. Li, S.W. Inst Physics, PEOPLE'S REPUBLIC OF CHINA

Prof. J.A.C. Cabral, Instituto Superior Tecnico, PORTUGAL

Prof. M.A. Hellberg, Univ. of Natal, S. AFRICA

Prof. D.E. Kim. Pohang Inst. of Sci. \& Tech., SO. KOREA

Prof. C.I.E.M.A.T, Fusion Division Library. SPAIN

Dr. L. Stenflo, Univ. of UMEA, SWEDEN

Library, Royal Inst. of Technology, SWEDEN

Prof. H. Wilhelmson, Chalmers Univ. of Tech., SWEDEN

Centre Phys. Des Plasmas, Ecole Polytech, SWITZERLAND

Bibliotheek, Inst. Voor Plasma-Fysica, THE NETHERLANDS

Asst. Prof. Dr. S. Cakir, Middle East Tech. Univ., TURKEY

Dr. V.A. Glukhikh,Sci. Res. Inst. Electrophys.I Apparatus, USSR

Dr. D.D. Ryutov, Siberian Branch of Academy of Sci., USSR

Dr. G.A. Eliseev, I.V. Kurchatov Inst., USSR

Librarian, The Ukr.SSR Academy of Sciences, USSR

Dr. L.M. Kovrizhnykh, Inst. of General Physics, USSR

Kemiorschungsanlage GmbH, Zentralbibliothek, W. GERMANY

Bibliothek, Inst. Für Plasmaforschung, W. GERMANY

Prof. K. Schindler, Ruhr-Universitát Bochum, W. GERMANY

Dr. F. Wagner, (ASDEX), Max-Planck-Institut, W. GERMANY

Librarian, Max-Planck-Institut, W. GERMANY 